\title{
A Conservative Gradient Discretization Method for Parabolic Equations
}

\author{
Huifang Zhou ${ }^{1}$, Zhiqiang Sheng ${ }^{2}$ and Guangwei Yuan ${ }^{2, *}$ \\ ${ }^{1}$ The Graduate School of China Academy of Engineering Physics, Beijing, China \\ ${ }^{2}$ Laboratory of Computational Physics, Institute of Applied Physics and Computational \\ Mathematics, Beijing, China
}

Received 22 February 2020; Accepted (in revised version) 9 May 2020

\begin{abstract}
In this paper, we propose a new conservative gradient discretization method (GDM) for one-dimensional parabolic partial differential equations (PDEs). We use the implicit Euler method for the temporal discretization and conservative gradient discretization method for spatial discretization. The method is based on a new cellcentered meshes, and it is locally conservative. It has smaller truncation error than the classical finite volume method on uniform meshes. We use the framework of the gradient discretization method to analyze the stability and convergence. The numerical experiments show that the new method has second-order convergence. Moreover, it is more accurate than the classical finite volume method in flux error, $L^{2}$ error and $L^{\infty}$ error.
\end{abstract}

AMS subject classifications: $65 \mathrm{M} 08,35 \mathrm{~K} 10$

Key words: Gradient discretization method, mass conservation, parabolic equations.

\section{Introduction}

Parabolic equations are a typical type of time-dependent problems. Many timedependent physical processes, such as heat conduction problems, underground engineering, oil recovery, and nuclear waste disposal, image analysis, can be described by parabolic equations $[11,12,15,22]$. Many numerical methods have been applied to parabolic equations, such as finite difference methods $[2,32]$, finite element methods $[17,31,37,40,41]$, discontinuous Galerkin methods [6, 29, 38], spectral Galerkin method [36], weak Galerkin method [39], and so on.

However, conventional numerical methods usually do not have local mass conservation property. Mass conservation property is crucial physically, and a numerical scheme

*Corresponding author.

Emails: 13614405274@163.com (H. Zhou), sheng_zhiqiang@iapcm.ac.cn (Z. Sheng),yuan_guangwei@ iapcm.ac.cn (G. Yuan) 
may produce a non-physical solution without local mass conservation. There have been a lot of efforts dedicated to the research of mass conservation preserving schemes. Different types of finite volume schemes have been proposed for the elliptic and parabolic equations. An implicit multi-point flux approximation on quadrilateral meshes is discussed in [1]. In [28], a minimal stencil finite volume scheme is studied. A monotone finite volume method is introduced in [35]. For the conservative finite difference methods, they also have been applied to various PDEs, including semilinear parabolic equation [10], hyperbolic conservation laws [9, 18], Helmholtz problem [23], acoustic wave equations [24,25], the kinetic and fluid simulations [33], multi-component flow computations, and transport process [30]. In recent years, high order conservative discontinuous Galerkin methods are also proposed for many PDEs, such as Klein-Gordon-Schrödinger equations [4], nonlinear electromagnetic Schrödinger equations [34], radiative transfer equations [26], and hyperbolic conservative equations [5,27].

The gradient discretization method (GDM) is an efficient numerical method for solving linear and nonlinear elliptic and parabolic partial differential equations, see [13]. The main idea of the GDM is to use discrete spaces and discrete differential operators to mimic the original continuous spaces and differential operators in a variational formulation. In fact, the gradient discretization method is a highly flexible framework consisting of a large family numerical methods, such as conforming finite element method, nonconforming finite element method, and two-point flux finite volume method. In recent years, many numerical methods are analyzed under the framework of GDM, including the vertex approximate gradient (VAG) methods [20,21], multi-point flux approximation method [1], hybrid mimetic mixed methods [14,15], nodal mimetic finite difference methods $[3,14]$, discrete duality finite volume methods $[7,8,16]$, and the nine-point stencil finite volume method [22].

In this paper, we propose a conservative gradient discretization method for 1-d parabolic equation, and analyze the stability and convergence of the scheme under the framework of GDM. The main advantages of this scheme are

- it has only cell-centered unknowns;

- the stencil only includes neighbor cells;

- it can be applied to general non-uniform meshes;

- it is locally conservative;

- the resulting linear system is symmetric and positive definite;

- the diffusion coefficient can be discontinuous or nonlinear;

- for linear cases, the convergence orders in $H^{1}$ and $L^{2}$ norms are derived; for quasilinear cases, the convergences of $H^{1}$ and $L^{2}$ norms are proved; 
- on uniform meshes, it has smaller truncation error than the classical finite volume scheme [19], and numerical experiments show that it is more accurate than the classical finite volume scheme.

The rest of paper is organized as follows. In Section 2, we propose the new conservative gradient discretization scheme. Its stability and convergence are proved in Section 3. In Section 4, the convergence order for discontinuous coefficients case is proved. In Section 5, the truncation errors of the new scheme and classical finite volume method are analyzed. Some numerical experiments are presented in Section 6. Finally, a brief summary is given in Section 7.

\section{The conservative gradient discretization scheme}

Consider the following diffusion problem

$$
\begin{array}{ll}
u_{t}(x, t)-\left(\kappa(x, t) u_{x}(x, t)\right)_{x}=f(x, t) & \text { in } Q_{T}=(0, l) \times(0, T], \\
u(0, t)=u(l, t)=0 & \text { in }[0, T], \\
u(x, 0)=u^{0}(x) & \text { in } Q=[0, l],
\end{array}
$$

where $f(x, t) \in L^{2}\left(Q_{T}\right), u^{0}(x) \in L^{2}(Q)$ and there exist positive constants $\sigma_{0}$ and $\sigma_{1}$ such that the diffusion coefficient satisfies $\sigma_{0} \leq \kappa(x, t) \leq \sigma_{1}$.

The weak formulation of this problem is: Find $u(x, t) \in L^{2}\left((0, T) ; H_{0}^{1}(Q)\right)$ such that

$$
\begin{aligned}
& -\int_{0}^{T} \int_{0}^{l} u(x, t) v_{t}(x, t) \mathrm{d} x \mathrm{~d} t-\int_{0}^{l} u^{0}(x) v(x, 0) \mathrm{d} x+\int_{0}^{T} \int_{0}^{l} \kappa(x, t) u_{x}(x, t) v_{x}(x, t) \mathrm{d} x \mathrm{~d} t \\
= & \int_{0}^{T} \int_{0}^{l} f(x, t) v(x, t) \mathrm{d} x \mathrm{~d} t,
\end{aligned}
$$

for all $v(x, t) \in L^{2}\left((0, T) ; H_{0}^{1}(Q)\right) \cap H^{1}\left(0, T ; L^{2}(Q)\right)$ satisfying $v(x, T)=0$.

\subsection{Notations}

The domain $Q=(0, l)$ is partitioned into the following non-uniform meshes:

$$
x_{-\frac{1}{2}}=0<x_{0}<x_{1}<\cdots<x_{J}<l=x_{J+\frac{1}{2}}
$$

where $x_{j}$ denotes the vertex for $0 \leq j \leq J, x_{j+\frac{1}{2}}$ denotes the center of cell $I_{j+\frac{1}{2}}=\left(x_{j}, x_{j+1}\right)$ satisfying $x_{j+\frac{1}{2}}=\left(x_{j}+x_{j+1}\right) / 2$ for all $0 \leq j \leq J-1, I_{-\frac{1}{2}}=\left(x_{-\frac{1}{2}}, x_{0}\right)$ and $I_{J+\frac{1}{2}}=\left(x_{J}, x_{J+\frac{1}{2}}\right)$. $x_{-\frac{1}{2}}$ and $x_{J+\frac{1}{2}}$ are the left and right boundaries of $Q$, which are viewed as cell-centers of boundary cells $I_{-\frac{1}{2}}$ and $I_{J+\frac{1}{2}}$, respectively. We define $h_{j+\frac{1}{2}}$ as the length of cell $I_{j+\frac{1}{2}}$ and define $h_{j}$ as the length of dual cell $I_{j}=\left(x_{j-\frac{1}{2}}, x_{j+\frac{1}{2}}\right)$.

$$
h=\max _{-1 \leq j \leq J} h_{j+\frac{1}{2}}
$$




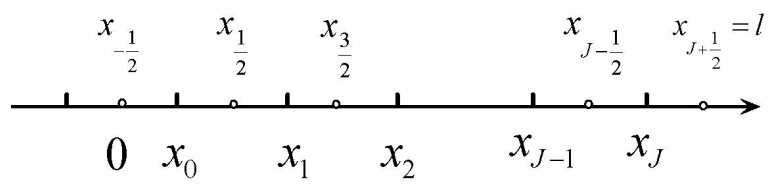

Figure 1: The Meshes.

denotes the mesh size. The figure of partition is shown in Fig. 1. Note that the meshes produced by this partition that are different from that of classical finite volume method [19]. The difference is that there are two half-cells on the boundary of $Q$, which are $I_{-\frac{1}{2}}$ and $I_{J+\frac{1}{2}}$, respectively. Throughout this paper, we use $C$ to denote generic constant irrelevant to mesh size $h$.

\subsection{Algorithm}

Now we begin to construct the conservative gradient discretization scheme for Eqs. (2.1a)-(2.1c). We use implicit Euler discretization and integrate the diffusion term on cell $I_{j+\frac{1}{2}}$. Denote

$$
U_{j+\frac{1}{2}}^{n}=\frac{1}{h_{j+\frac{1}{2}}} \int_{x_{j}}^{x_{j+1}} u\left(x, t^{n}\right) \mathrm{d} x
$$

We have

$$
\int_{x_{j}}^{x_{j+1}} u_{t}\left(x, t^{n+1}\right) \mathrm{d} x \approx h_{j+\frac{1}{2}} \frac{U_{j+\frac{1}{2}}^{n+1}-U_{j+\frac{1}{2}}^{n}}{\tau} .
$$

Denote the flux $F(x, t)=-\kappa u_{x}$, where $\kappa=\kappa(x, t)$ is piecewise smooth, with discontinuity at certain cell-vertex. By using the formula of the integration by parts, we have

$$
-\left.\int_{x_{j}}^{x_{j+1}}\left(\kappa u_{x}\right)_{x}\right|_{t=t^{n+1}} \mathrm{~d} x=F\left(x_{j+1}, t^{n+1}\right)-F\left(x_{j}, t^{n+1}\right) \approx F_{j+1}^{n+1}-F_{j}^{n+1},
$$

where $F_{j}^{n+1}$ is the approximation of $F\left(x_{j}, t^{n+1}\right)$. From (2.3) and (2.4), the discretization of Eq. (2.1a) is

$$
\frac{U_{j+\frac{1}{2}}^{n+1}-U_{j+\frac{1}{2}}^{n}}{\tau}+\frac{1}{h_{j+\frac{1}{2}}}\left(F_{j+1}^{n+1}-F_{j}^{n+1}\right)=f_{j+\frac{1}{2}}^{n+1}
$$

where

$$
f_{j+\frac{1}{2}}^{n+1}=\frac{1}{h_{j+\frac{1}{2}}} \int_{x_{j}}^{x_{j+1}} f\left(x, t^{n+1}\right) \mathrm{d} x .
$$

Then, we need to define the numerical flux $F_{j}^{n+1}$. 
First, we define the discrete unilateral flux

$$
\begin{aligned}
& F_{j}^{n+1,+}=-\kappa_{j+\frac{1}{2}}^{n+1} \frac{U_{j+\frac{1}{2}}^{n+1}-U_{j}^{n+1}}{\frac{h_{j+\frac{1}{2}}}{2}}, \\
& F_{j}^{n+1,-}=-\kappa_{j-\frac{1}{2}}^{n+1} \frac{U_{j}^{n+1}-U_{j-\frac{1}{2}}^{n+1}}{\frac{h_{j-\frac{1}{2}}}{2}},
\end{aligned}
$$

where

$$
\kappa_{j+\frac{1}{2}}^{n+1}=\kappa\left(x_{j+\frac{1}{2}}, t^{n+1}\right)
$$

We require the continuity of flux, i.e.,

$$
F_{j}^{n+1,+}=F_{j}^{n+1,-},
$$

then we obtain

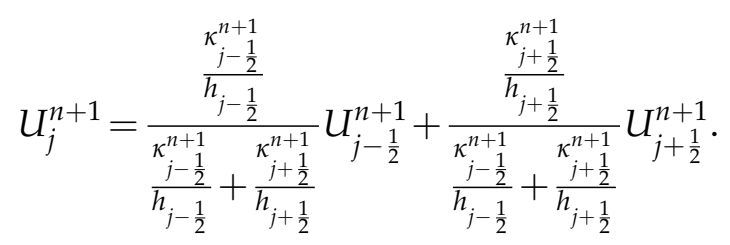

Define the conservative numerical flux $F_{j}^{n+1}=F_{j}^{n+1,+}=F_{j}^{n+1,-}$. Substituting (2.6) into (2.5a) yields the numerical flux

$$
F_{j}^{n+1}=-\kappa_{j}^{n+1} \frac{U_{j+\frac{1}{2}}^{n+1}-U_{j-\frac{1}{2}}^{n+1}}{h_{j}},
$$

where

$$
\kappa_{j}^{n+1}=\frac{2{\frac{\kappa_{j-\frac{1}{2}}^{n+1}}{h_{j-\frac{1}{2}}} \kappa_{j+\frac{1}{2}}^{n+1}}_{h_{j+\frac{1}{2}}}^{\kappa_{j-\frac{1}{2}}^{n+1}}+\kappa_{j+\frac{1}{2}}^{n+1}}{h_{j-\frac{1}{2}}} .
$$

Hence we obtain the conservative gradient discretization scheme of (2.1a)-(2.1c) as follows:

Algorithm 2.1 (The gradient discretization scheme). For any $0 \leq j \leq J-1$ and $0 \leq n \leq N-1$,

$$
\begin{aligned}
& \frac{U_{j+\frac{1}{2}}^{n+1}-U_{j+\frac{1}{2}}^{n}}{\tau}+\frac{1}{h_{j+\frac{1}{2}}}\left(F_{j+1}^{n+1}-F_{j}^{n+1}\right)=f_{j+\frac{1}{2}}^{n+1}, \\
& U_{-\frac{1}{2}}^{n+1}=U_{J+\frac{1}{2}}^{n+1}=0, \\
& U_{j+\frac{1}{2}}^{0}=u_{j+\frac{1}{2}}^{0}{ }^{\prime}
\end{aligned}
$$


where

$$
f_{j+\frac{1}{2}}^{n+1}=\frac{1}{h_{j+\frac{1}{2}}} \int_{x_{j}}^{x_{j+1}} f\left(x, t^{n+1}\right) \mathrm{d} x, \quad u_{j+\frac{1}{2}}^{0}=\frac{1}{h_{j+\frac{1}{2}}} \int_{x_{j}}^{x_{j+1}} u^{0}(x) \mathrm{d} x .
$$

Remark 2.1. It should be noticed that the main difference between the conservative gradient discretization scheme (2.7a)-(2.7b) and the finite volume scheme in [19] is that they use different meshes, which leads to different construction of flux on boundary cells. The detailed comparison can be found in Section 5 .

Remark 2.2. Many numerical schemes can be encompassed in the GDM framework, such as the conforming finite element method has different discrete reconstruction operator, the discontinuous Galerkin method has different discrete space and discrete gradient, and the hybrid mimetic mixed method has different discrete space. However, they are different from the conservative gradient discretization scheme proposed in (2.7a)-(2.7b). The further illustration of these methods can be found in [13].

\subsection{Discrete variational form}

The GDM in [13] consists of three key components: discrete space, discrete reconstruction operator, and the discrete gradient. We rewrite the conservative gradient discretization scheme (2.7a)-(2.7b) into variational form. For this, we need to introduce the following discrete space $X_{D, 0}$, and the discrete reconstruction operators.

- We define $X_{D, 0}$ as the set of cell-centered unknowns $U^{n+1}=\left\{U_{i+\frac{1}{2}}^{n+1}\right\}_{i=-1}^{J}$ for $0 \leq n \leq N$ satisfying Diriclet boundary condition (2.1b), i.e., $U_{-\frac{1}{2}}^{n+1}=U_{J+\frac{1}{2}}^{n+1}=0$.

- For any $U^{n+1} \in X_{D, 0}$, We define $\Pi_{D} U^{n+1}$ a piecewise constant function being equal to $U_{j+\frac{1}{2}}^{n+1}$ on each cell $I_{j+\frac{1}{2}}$.

- The gradient $\delta_{h} U^{n+1}$ is constructed to be a piecewise constant function being equal to $\frac{U_{j+\frac{1}{2}}^{n+1}-U_{j-\frac{1}{2}}^{n+1}}{h_{j}}$ on each dual cell $I_{j}$.

- For the discrete unknowns $U^{0}, U^{1}, \cdots, U^{N} \in X_{D, 0}, \delta_{t} U^{k+1}$ are piecewise constant functions defined by $\left(\Pi_{D} U^{k+1}-\Pi_{D} U^{k}\right) / \tau$, for any $0 \leq k \leq N-1$.

Before rewriting the variational form of conservative gradient discretization scheme, we need to introduce the discrete formula of integration by parts.

Lemma 2.1 (Discrete formula of integration by parts). For discrete unknowns $V \in X_{D, 0}$ and $\left\{W_{j} \mid j=0,1, \cdots, J\right\}$, it holds that

$$
\sum_{j=0}^{J-1}\left(W_{j+1}-W_{j}\right) V_{j+\frac{1}{2}}=-\sum_{j=0}^{J}\left(V_{j+\frac{1}{2}}-V_{j-\frac{1}{2}}\right) W_{j} .
$$


We take $V \in X_{D, 0}$. Multiplying (2.7a) by $V_{j+\frac{1}{2}}^{n+1} h_{j+\frac{1}{2}}$ and summing over $j=0,1, \cdots, J-1$, from Lemma 2.1 we obtain

$$
\begin{aligned}
& \int_{0}^{l} f\left(x, t^{n+1}\right) \Pi_{D} V_{D}(x) \mathrm{d} x=\sum_{j=0}^{J-1} f_{j+\frac{1}{2}}^{n+1} V_{j+\frac{1}{2}} h_{j+\frac{1}{2}} \\
= & \sum_{j=0}^{J-1} \frac{U_{j+\frac{1}{2}}^{n+1}-U_{j+\frac{1}{2}}^{n}}{\tau} V_{j+\frac{1}{2}} h_{j+\frac{1}{2}}-\sum_{j=0}^{J-1}\left(\kappa_{j+1}^{n+1} \frac{\left.U_{j+\frac{3}{2}}^{n+1}-U_{j+\frac{1}{2}}^{n+1}-\kappa_{j}^{n+1} \frac{U_{j+\frac{1}{2}}^{n+1}-U_{j-\frac{1}{2}}^{n+1}}{h_{j+1}}\right) V_{j+\frac{1}{2}}}{h_{j}}\right. \\
= & \sum_{j=0}^{J-1} \frac{U_{j+\frac{1}{2}}^{n+1}-U_{j+\frac{1}{2}}^{n}}{\tau} V_{j+\frac{1}{2}} h_{j+\frac{1}{2}}+\sum_{j=0}^{J} \kappa_{j}^{n+1} \frac{U_{j+\frac{1}{2}}^{n+1}-U_{j-\frac{1}{2}}^{n+1}}{h_{j}}\left(V_{j+\frac{1}{2}}-V_{j-\frac{1}{2}}\right) \\
= & \int_{0}^{l} \delta_{t} U^{n+1}(x) V(x) \mathrm{d} x+\int_{0}^{l} \delta_{h} U^{n+1}(x) \delta_{h} V(x) \mathrm{d} x .
\end{aligned}
$$

Finally, the conservative gradient discretization scheme can be written into the following discrete variational problem:

Find a family of $\left\{U^{n+1}\right\}_{n=0}^{N-1} \in X_{D, 0}$ such that, for all $V \in X_{D, 0}$, there are $U^{0}=\left\{u_{j+\frac{1}{2}}^{0}\right\}_{j=-1}^{J}$ and

$$
\begin{aligned}
& \int_{0}^{l} \delta_{t} U^{n+1}(x) \Pi_{D} V(x) \mathrm{d} x+\int_{0}^{l} \delta_{h} U^{n+1}(x) \delta_{h} V(x) \mathrm{d} x \\
= & \int_{0}^{l} f\left(x, t^{n+1}\right) \Pi_{D} V_{D}(x) \mathrm{d} x .
\end{aligned}
$$

Remark 2.3. It is easy to see that the discrete variational problem is equivalent to the conservative gradient discretization scheme (2.7a)-(2.7b).

\section{Stability and convergence}

In this section, we follow the argument framework of gradient discretization method in [13] to prove the stability and convergence of the gradient discretization scheme (2.7a)(2.7b). The main idea of the gradient discretization method is to use discrete spaces and discrete differential operators to mimic the original continuous spaces and differential operators in the variational formulation.

\subsection{Stability analysis}

Now we define the discrete $L^{2}$ norms,

$$
\|U\|_{d}^{2}=\sum_{i=0}^{J-1} U_{i+\frac{1}{2}}^{2} h_{i+\frac{1}{2}}
$$




$$
\left\|\delta_{h} U\right\|_{d}^{2}=\sum_{i=0}^{J} \frac{\left(U_{i+\frac{1}{2}}-U_{i-\frac{1}{2}}\right)^{2}}{h_{i}} .
$$

For any $1 \leq k \leq N-1$, we also denote $\left\|f^{k+1}\right\|_{d}$ the discrete $L^{2}$ norm for $\left\{f_{i+\frac{1}{2}}^{k+1}\right\}_{i=0}^{J-1}$, i.e.,

$$
\left\|f^{k+1}\right\|_{d}^{2}=\sum_{i=0}^{J-1}\left(f_{i+\frac{1}{2}}^{k+1}\right)^{2} h_{i+\frac{1}{2}} .
$$

It should be noticed that $\left\|f^{k+1}\right\|_{d}$ is the discrete $L^{2}$ norm for $\left\{f_{i+\frac{1}{2}}^{k+1}\right\}_{i=0}^{J-1}$ instead of the $L^{2}$ norm of $f^{k+1}$.

Under these discrete $L^{2}$ norms, we have the following discrete Poincare's inequality, which plays an essential role in the convergence analysis. The proof is left to interested readers.

Lemma 3.1 (Discrete Poincare's inequality). For any $U \in X_{D, 0}$, there holds

$$
\|U\|_{d} \leq l\left\|\delta_{h} U\right\|_{d}
$$

where $l$ is the length of domain $Q$.

In the following theorem, we prove the stability of the conservative gradient discretization scheme (2.7a)-(2.7b).

Theorem 3.1. The solution of scheme (2.7a)-(2.7b) satisfies

$$
\left\|U^{n+1}\right\|_{d}^{2} \leq\left\|U^{0}\right\|_{d}^{2}+\tau l^{2} \sum_{m=1}^{n+1}\left\|f^{m+1}\right\|_{d}^{2}
$$

Proof. Taking $V=U^{n+1}$ in the discrete variational problem (2.9), according to Lemma 3.1, we get

$$
\begin{aligned}
& \int_{0}^{l} \delta_{t} U^{n+1}(x) \Pi_{D} U^{n+1}(x) \mathrm{d} x+\int_{0}^{l} \delta_{h} U^{n+1}(x)^{2} \mathrm{~d} x \\
= & \frac{1}{2 \tau}\left(\left\|U^{n+1}\right\|_{d}^{2}-\left\|U^{n}\right\|_{d}^{2}+\left\|U^{n+1}-U^{n}\right\|_{d}^{2}\right)+\left\|\delta_{h} U^{n+1}\right\|_{d}^{2} \\
\geq & \frac{1}{2 \tau}\left(\left\|U^{n+1}\right\|_{d}^{2}-\left\|U^{n}\right\|_{d}^{2}+\left\|U^{n+1}-U^{n}\right\|_{d}^{2}\right)+\frac{1}{l^{2}}\left\|U^{n+1}\right\|_{d}^{2} .
\end{aligned}
$$

According to Cauchy inequality $a b \leq \varepsilon a^{2}+\frac{1}{4 \varepsilon} b^{2}$, we have

$$
\sum_{j=0}^{J-1} f_{j+\frac{1}{2}}^{n+1} U_{j+\frac{1}{2}}^{n+1} h_{j+\frac{1}{2}} \leq \varepsilon\left\|U^{n+1}\right\|_{d}^{2}+\frac{1}{4 \varepsilon}\left\|f^{n+1}\right\|_{d}^{2} .
$$


Taking $\varepsilon=\frac{1}{2 l^{2}}$, there is

$$
\left\|U^{n+1}\right\|_{d}^{2}-\left\|U^{n}\right\|_{d}^{2} \leq \tau l^{2}\left\|f^{n+1}\right\|_{d}^{2}
$$

By conduction, we can come to the conclusion

$$
\left\|U^{n+1}\right\|_{d}^{2} \leq\left\|U^{0}\right\|_{d}^{2}+\tau l^{2} \sum_{m=1}^{n+1}\left\|f^{m+1}\right\|_{d}^{2}
$$

Thus, we complete the proof.

\subsection{Convergence analysis}

According to the framework in [13], in order to get the convergence of scheme (2.9) we need to verify the GD-consistency and the limit-conformity.

Suppose the exact solution $u \in L^{\infty}\left(0, T ; W_{s}\right)$, where $W_{s}=H^{2}(Q) \cap H_{0}^{1}(Q)$. First we introduce the definition of GD-consistency.

Definition 3.1 (GD-consistency). Define $\mathcal{S}_{\mathcal{D}}: W_{s} \rightarrow[0, \infty)$ by

$$
\mathcal{S}_{\mathcal{D}}(\varphi)=\min _{V \in X_{D, 0}}\left(\left\|\Pi_{D} V-\varphi\right\|_{L^{2}(Q)}+\left\|\delta_{h} V-\varphi^{\prime}\right\|_{L^{2}(Q)}\right), \quad \forall \varphi \in W_{s} .
$$

A discretization is GD-consistent if

$$
\lim _{h \rightarrow 0} \mathcal{S}_{\mathcal{D}}(\varphi)=0, \quad \forall \varphi \in W_{s} .
$$

Lemma 3.2 (GD-consisitency). The discretization (2.9) is GD-consistent. Moreover, it holds that

$$
\mathcal{S}_{\mathcal{D}}(\varphi) \leq C h\|\varphi\|_{H^{2}(Q)}, \quad \forall \varphi \in W_{s} .
$$

Proof. In the definition of GD-consistent (3.2), we select

$$
V=\left(\varphi\left(x_{-\frac{1}{2}}\right), \varphi\left(x_{\frac{1}{2}}\right), \cdots, \varphi\left(x_{J+\frac{1}{2}}\right)\right),
$$

and estimate the two terms $\left\|\Pi_{D} V-\varphi\right\|_{L^{2}(Q)}$ and $\left\|\delta_{h} V-\varphi^{\prime}\right\|_{L^{2}(Q)}$, separately.

For the first term, on each interval $\left(x_{j}, x_{j+1}\right), j=1, \cdots, J-1$, we have

$$
\int_{x_{j}}^{x_{j+1}}\left(\Pi_{D} V(x)-\varphi(x)\right)^{2} \mathrm{~d} x=\int_{x_{j}}^{x_{j+1}}\left(\int_{x_{j+\frac{1}{2}}}^{x} \varphi^{\prime}(y) \mathrm{d} y\right)^{2} \mathrm{~d} x .
$$


From the Cauchy-Schwarz inequality, we have

$$
\begin{aligned}
& \int_{x_{j}}^{x_{j+1}}\left(\Pi_{D} V(x)-\varphi(x)\right)^{2} \mathrm{~d} x \\
\leq & \int_{x_{j}}^{x_{j+1}} \int_{x_{j+\frac{1}{2}}}^{x}\left(\varphi^{\prime}(y)\right)^{2} \mathrm{~d} y\left(x-x_{j+\frac{1}{2}}\right) \mathrm{d} x \\
\leq & \int_{x_{j}}^{x_{j+1}} \int_{x_{j}}^{x_{j+1}}\left(\varphi^{\prime}(y)\right)^{2} \mathrm{~d} y h_{j+\frac{1}{2}} \mathrm{~d} x \\
= & h_{j+\frac{1}{2}}^{2} \int_{x_{j}}^{x_{j+1}}\left(\varphi^{\prime}(x)\right)^{2} \mathrm{~d} x .
\end{aligned}
$$

Similarly, on $\left(x_{-\frac{1}{2}}, x_{0}\right)$ and $\left(x_{J}, x_{J+\frac{1}{2}}\right)$ we have

$$
\begin{aligned}
& \int_{x_{-\frac{1}{2}}}^{x_{0}}\left(\Pi_{D} V(x)-\varphi(x)\right)^{2} \mathrm{~d} x \leq h_{-\frac{1}{2}}^{2} \int_{x_{-\frac{1}{2}}}^{x_{0}}\left(\varphi^{\prime}(x)\right)^{2} \mathrm{~d} x, \\
& \int_{x_{J}}^{x_{J+\frac{1}{2}}}\left(\Pi_{D} V(x)-\varphi(x)\right)^{2} \mathrm{~d} x \leq h_{J+\frac{1}{2}}^{2} \int_{x_{J}}^{x_{J+\frac{1}{2}}}\left(\varphi^{\prime}(x)\right)^{2} \mathrm{~d} x .
\end{aligned}
$$

Summing (3.4) over $j=1, \cdots, J-1$, (3.5a) and (3.5b) yields

$$
\left\|\Pi_{D} V-\varphi\right\|_{L^{2}(Q)}^{2} \leq h^{2}|\varphi|_{H^{1}(Q)}^{2} .
$$

For the second term, on each interval $\left(x_{j-\frac{1}{2}}, x_{j+\frac{1}{2}}\right), j=0, \cdots, J$, from the definition of $\delta_{h} V$, we have

$$
\begin{aligned}
& \int_{x_{j-\frac{1}{2}}}^{x_{j+\frac{1}{2}}}\left(\delta_{h} V(x)-\varphi^{\prime}(x)\right)^{2} \mathrm{~d} x \\
= & \int_{x_{j-\frac{1}{2}}}^{x_{j+\frac{1}{2}}}\left(\frac{1}{h_{j}}\left(\varphi\left(x_{j+\frac{1}{2}}\right)-\varphi\left(x_{j-\frac{1}{2}}\right)\right)-\varphi^{\prime}(x)\right)^{2} \mathrm{~d} x \\
= & \int_{x_{j-\frac{1}{2}}}^{x_{j+\frac{1}{2}}}\left(\frac{1}{h_{j}} \int_{x_{j-\frac{1}{2}}}^{x_{j+\frac{1}{2}}}\left(\varphi^{\prime}(y)-\varphi^{\prime}(x)\right) \mathrm{d} y\right)^{2} \mathrm{~d} x \\
= & \frac{1}{h_{j}^{2}} \int_{x_{j-\frac{1}{2}}}^{x_{j+\frac{1}{2}}}\left(\int_{x_{j-\frac{1}{2}}}^{x_{j+\frac{1}{2}}} \int_{y}^{x} \varphi^{\prime \prime}(z) \mathrm{d} z \mathrm{~d} y\right)^{2} \mathrm{~d} x .
\end{aligned}
$$

By using the Cauchy-Schwarz inequality twice, we obtain

$$
\begin{aligned}
& \int_{x_{j-\frac{1}{2}}}^{x_{j+\frac{1}{2}}}\left(\delta_{h} V(x)-\varphi^{\prime}(x)\right)^{2} \mathrm{~d} x \\
\leq & \frac{1}{h_{j}} \int_{x_{j-\frac{1}{2}}}^{x_{j+\frac{1}{2}}} \int_{x_{j-\frac{1}{2}}}^{x_{j+\frac{1}{2}}}\left(\int_{y}^{x} \varphi^{\prime \prime}(z) \mathrm{d} z\right)^{2} \mathrm{~d} y \mathrm{~d} x
\end{aligned}
$$




$$
\begin{aligned}
& \leq \frac{1}{h_{j}} \int_{x_{j-\frac{1}{2}}}^{x_{j+\frac{1}{2}}} \int_{x_{j-\frac{1}{2}}}^{x_{j+\frac{1}{2}}} \int_{y}^{x}\left(\varphi^{\prime \prime}(z)\right)^{2} \mathrm{~d} z(t-x) \mathrm{d} y \mathrm{~d} x \\
& \leq \int_{x_{j-\frac{1}{2}}}^{x_{j+\frac{1}{2}}} \int_{x_{j-\frac{1}{2}}}^{x_{j+\frac{1}{2}}} \int_{x_{j-\frac{1}{2}}}^{x_{j+\frac{1}{2}}}\left(\varphi^{\prime \prime}(z)\right)^{2} \mathrm{~d} z \mathrm{~d} y \mathrm{~d} x \\
& =h_{j}^{2} \int_{x_{j-\frac{1}{2}}}^{x_{j+\frac{1}{2}}}\left(\varphi^{\prime \prime}(x)\right)^{2} \mathrm{~d} x .
\end{aligned}
$$

Summing (3.7) over $j=0, \cdots, J$ yields

$$
\left\|\delta_{h} V-\varphi^{\prime}\right\|_{L^{2}(Q)}^{2} \leq h^{2}|\varphi|_{H^{2}(Q)}^{2} .
$$

Combing (3.6) and (3.8), we complete the proof.

Suppose the derivative of exact solution $u^{\prime} \in L^{\infty}\left(0, T ; W_{w}\right)$, where $W_{w}=H^{1}(Q)$. We introduce the limit-conforming property.

Definition 3.2 (Limit-conforming). Define $W_{D}: W_{w} \rightarrow[0, \infty)$ by

$$
W_{D}(\varphi)=\sup _{V \in X_{D, 0} \backslash\{0\}} \frac{\left|\int_{Q}\left(\delta_{h} V(x) \varphi(x)+\Pi_{D} V(x) \varphi^{\prime}(x)\right) \mathrm{d} x\right|}{\left\|\delta_{h} V\right\|_{d}}, \forall \varphi \in W_{w} .
$$

A discretization is limit-conforming if

$$
\lim _{h \rightarrow 0} W_{D}(\varphi)=0, \quad \forall \varphi \in W_{w} .
$$

Lemma 3.3 (Limit-conforming). The discretization (2.9) is limit-conforming. Moreover, it holds that

$$
W_{D}(\varphi) \leq h\|\varphi\|_{H^{1}(Q)}, \quad \forall \varphi \in W_{w} .
$$

Proof. From the definition of $\delta_{h} V$ we have

$$
\begin{aligned}
& \int_{Q} \delta_{h} V(x) \varphi(x) \mathrm{d} x \\
= & \sum_{j=0}^{J} \int_{x_{j-\frac{1}{2}}}^{x_{j+\frac{1}{2}}} \frac{1}{h_{j}}\left(V_{j+\frac{1}{2}}-V_{j-\frac{1}{2}}\right) \varphi(x) \mathrm{d} x \\
= & \sum_{j=0}^{J}\left(V_{j+\frac{1}{2}}-V_{j-\frac{1}{2}}\right) \bar{\varphi}_{j},
\end{aligned}
$$

where

$$
\bar{\varphi}_{j}=\frac{1}{h_{j}} \int_{x_{j-\frac{1}{2}}}^{x_{j+\frac{1}{2}}} \varphi(x) \mathrm{d} x
$$


From the definition of $\Pi_{D}$ and the boundary condition $V_{-\frac{1}{2}}=V_{J+\frac{1}{2}}=0$, we have

$$
\begin{aligned}
& \int_{Q} \Pi_{D} V(x) \varphi^{\prime}(x) \mathrm{d} x \\
= & \sum_{j=0}^{J-1} \int_{x_{j}}^{x_{j+1}} V_{j+\frac{1}{2}} \varphi^{\prime}(x) \mathrm{d} x \\
= & \sum_{j=0}^{J-1} V_{j+\frac{1}{2}}\left(\varphi\left(x_{j+1}\right)-\varphi\left(x_{j}\right)\right) .
\end{aligned}
$$

From the discrete integration by parts in Lemma 2.1, we have

$$
\int_{Q} \Pi_{D} V(x) \varphi^{\prime}(x) \mathrm{d} x=-\sum_{j=0}^{J}\left(V_{j+\frac{1}{2}}-V_{j-\frac{1}{2}}\right) \varphi\left(x_{j}\right) .
$$

Summing (3.11) and (3.12) and using the Cauchy-Schwarz inequality, we obtain

$$
\begin{aligned}
& \int_{Q}\left(\delta_{h} V(x) \varphi(x)+\Pi_{D} V(x) \varphi^{\prime}(x)\right) \mathrm{d} x \\
= & \sum_{j=0}^{J}\left(V_{j+\frac{1}{2}}-V_{j-\frac{1}{2}}\right)\left(\bar{\varphi}_{j}-\varphi\left(x_{j}\right)\right) \\
\leq & \left(\sum_{j=0}^{J} \frac{\left(V_{j+\frac{1}{2}}-V_{j-\frac{1}{2}}\right)^{2}}{h_{j}}\right)^{\frac{1}{2}}\left(\sum_{j=0}^{J} h_{j}\left(\bar{\varphi}_{j}-\varphi\left(x_{j}\right)\right)^{2}\right)^{\frac{1}{2}} \\
= & \left\|\delta_{h} V\right\|_{d}\left(\sum_{j=0}^{J} h_{j}\left(\bar{\varphi}_{j}-\varphi\left(x_{j}\right)\right)^{2}\right)^{\frac{1}{2}},
\end{aligned}
$$

which implies

$$
W_{D}(\varphi) \leq\left(\sum_{j=0}^{J} h_{j}\left(\bar{\varphi}_{j}-\varphi\left(x_{j}\right)\right)^{2}\right)^{\frac{1}{2}} .
$$

From the Newton-Leibniz formula, we have

$$
\begin{aligned}
& \sum_{j=0}^{J} h_{j}\left(\bar{\varphi}_{j}-\varphi\left(x_{j}\right)\right)^{2} \\
= & \sum_{j=0}^{J} \frac{1}{h_{j}}\left(\int_{x_{j-\frac{1}{2}}}^{x_{j+\frac{1}{2}}} \varphi(x)-\varphi\left(x_{j}\right) \mathrm{d} x\right)^{2} \\
= & \sum_{j=0}^{J} \frac{1}{h_{j}}\left(\int_{x_{j-\frac{1}{2}}}^{x_{j+\frac{1}{2}}} \int_{x_{j}}^{x} \varphi^{\prime}(y) \mathrm{d} y \mathrm{~d} x\right)^{2} .
\end{aligned}
$$


Then by using the Cauchy-Schwarz inequality, we obtain

$$
\begin{aligned}
& \sum_{j=0}^{J} h_{j}\left(\bar{\varphi}_{j}-\varphi\left(x_{j}\right)\right)^{2} \\
\leq & \sum_{j=0}^{J} \int_{x_{j-\frac{1}{2}}}^{x_{j+\frac{1}{2}}}\left(\int_{x_{j}}^{x} \varphi^{\prime}(y) \mathrm{d} y\right)^{2} \mathrm{~d} x \\
\leq & \sum_{j=0}^{J} \int_{x_{j-\frac{1}{2}}}^{x_{j+\frac{1}{2}}} \int_{x_{j}}^{x}\left(\varphi^{\prime}(y)\right)^{2} \mathrm{~d} y\left(x-x_{j}\right) \mathrm{d} x \\
\leq & \sum_{j=0}^{J} \int_{x_{j-\frac{1}{2}}}^{x_{j+\frac{1}{2}}} \int_{x_{j-\frac{1}{2}}}^{x_{j+\frac{1}{2}}}\left(\varphi^{\prime}(y)\right)^{2} \mathrm{~d} y h_{j} \mathrm{~d} x \\
= & \sum_{j=0}^{J} h_{j}^{2} \int_{x_{j-\frac{1}{2}}}^{x_{j+\frac{1}{2}}}\left(\varphi^{\prime}(x)\right)^{2} \mathrm{~d} x \\
\leq & h^{2}|\varphi|_{H^{1}(Q)^{\prime}}^{2}
\end{aligned}
$$

which completes the proof.

Definition 3.3. The space size $h_{D}$ is defined by

$$
h_{D}\left(W_{s} ; W_{w}\right)=\max \left(s_{D}\left(W_{s}\right), w_{D}\left(W_{w}\right)\right),
$$

where

$$
\begin{aligned}
& s_{D}\left(W_{s}\right)=\sup \left\{\frac{\mathcal{S}_{\mathcal{D}}(\varphi)}{\|\varphi\|_{W_{s}}}: \varphi \in W_{s} \backslash\{0\}\right\}, \\
& w_{D}\left(W_{w}\right)=\sup \left\{\frac{\mathcal{W}_{\mathcal{D}}(\varphi)}{\|\varphi\|_{W_{w}}}: \varphi \in W_{w} \backslash\{0\}\right\} .
\end{aligned}
$$

From Lemma 3.2 and Lemma 3.3, we obtain that for the discretization (2.9), the space size is

$$
h_{D}\left(W_{s} ; W_{w}\right) \leq C h
$$

Denote the error of initial condition

$$
e^{i n i}=\left\|u^{0}-\Pi_{D} U^{0}\right\|_{L^{2}(Q)} .
$$

Similar to the previous proof, we have

$$
e^{i n i} \leq\left|u^{0}\right|_{H_{1}(Q)} h, \quad \forall u^{0} \in H_{0}^{1}(Q) .
$$

With the previous preparations, we obtain the following the error estimate theorems for the linear problem. The proof of Theorem 3.2 can be found in Theorem 5.3 in [13]. 
Theorem 3.2. Suppose $\kappa$ be a function bounded by $\sigma_{0} \leq \kappa \leq \sigma_{1}, f \in L^{2}\left(Q_{T}\right)$, and $u^{0} \in L^{2}(Q)$. Assume the exact solution $u$ is Lipschiz continuous $[0, T] \rightarrow W^{2, \infty}(Q)$ and $\left\{U^{n}\right\}_{n=1}^{N}$ are the solutions of (2.9). Denote $\tau$ the time step size, $h_{D}$ the space size, and $e^{i n i}$ the initial error, then there exists a constant $C$ such that

$$
\begin{aligned}
& \max _{n=1, \cdots, N}\left\|u\left(\cdot, t_{n}\right)-\Pi_{D} U^{n}\right\|_{L^{2}(Q)} \leq C\left(\tau+h_{D}+e^{i n i}\right), \\
& \left\|u^{\prime}-\delta_{h} U\right\|_{L^{2}\left(Q_{T}\right)} \leq C\left(\tau+h_{D}+e^{i n i}\right) .
\end{aligned}
$$

From (3.13) and (3.14), we have the following error estimate.

Theorem 3.3. Under the condition of Theorem 3.2 and $u^{0} \in H_{0}^{1}(Q)$, there exists a constant $C$ such that

$$
\begin{aligned}
& \max _{n=1, \cdots, N}\left\|u\left(\cdot, t_{n}\right)-\Pi_{D} U^{n}\right\|_{L^{2}(Q)} \leq C(\tau+h), \\
& \left\|u^{\prime}-\delta_{h} U\right\|_{L^{2}\left(Q_{T}\right)} \leq C(\tau+h) .
\end{aligned}
$$

Finally, we give the convergence result for the quasilinear problem. To prove the convergence we introduce the definitions of time consistent and compactness.

Definition 3.4 (Space-time-consistent). A discretization is space-time-consistent if

$$
\begin{array}{ll}
\lim _{h \rightarrow 0} \mathcal{S}_{\mathcal{D}}(\varphi)=0, & \forall \varphi \in W_{s}, \\
\lim _{h \rightarrow 0}\left\|v-\Pi_{D} I_{D} v\right\|_{L^{2}(Q)}=0, & \forall v \in L^{2}(Q) . \\
\tau \rightarrow 0 & \text { as } h \rightarrow 0 .
\end{array}
$$

Eqs. (3.15) is proved in Remark 4.4 of [13], hence the discretization (2.9) is space-timeconsistent.

To deal with the quasilinearity, we require the compactness property.

Definition 3.5 (Compactness). A discretization is compact if, for any sequence $\left\{U_{h}\right\} \in$ $X_{D, 0}$ such that $\left\{\left\|\delta_{h} U_{h}\right\|_{L^{2}(Q)}\right\}$ is bounded, the sequence $\left\{\Pi_{D} U_{h}\right\}$ is relatively compact in $L^{2}(Q)$.

According to discrete Poincare's inequality, the sequence $\left\{\left\|\Pi_{D} U_{h}\right\|_{L^{2}(Q)}\right\}$ is bounded when sequence $\left\{\left\|\delta_{h} U_{h}\right\|_{L^{2}(Q)}\right\}$ is bounded. The bounded set is relatively compact in finite dimensional space $X_{D, 0}$. Hence the discretization (2.9) is compact.

Finally, we state the convergence result for the quasilinear problem. The proof of this theorem can be found in Theorem 5.4 in [13].

Theorem 3.4. Suppose $\kappa$ be a function bounded by $\sigma_{0} \leq \kappa \leq \sigma_{1}, f \in L^{2}\left(Q_{T}\right)$, and $u^{0} \in L^{2}(Q)$. There hold

$$
\begin{aligned}
& \sup _{n \in[0, N]}\left\|\Pi_{D} U^{n}-u\left(\cdot, t^{n}\right)\right\|_{L^{2}(Q)} \rightarrow 0, \\
& \delta_{h} U^{n} \rightarrow u^{\prime}\left(\cdot, t^{n}\right) \quad \text { in } L^{2}\left(Q_{T}\right),
\end{aligned}
$$

where $u$ is the exact solution of problem (2.1a)-(2.1c). 
Remark 3.1. Since the convergence analysis follows the framework of GDM method in [13], the conditions and conclusions of Theorem 3.3 and Theorem 3.4 are same as Theorem 5.3 and Theorem 5.4 in [13], respectively.

\section{Discontinuous coefficients case}

Then we consider the discontinuous coefficients. First we introduce the Gronwall's inequality.

Lemma 4.1 (Discrete Gronwall's inequality). Suppose $f^{n} \geq 0,(n=0,1, \cdots, N)$ satisfy

$$
f^{n+1}-f^{n} \leq B \tau\left(f^{n+1}+f^{n}\right)+C_{n} \tau, \quad n=0,1, \cdots, N-1,
$$

where $B$ is a non-negative constant, $C_{n} \geq 0, N \tau=T$, then

$$
f^{n+1} \leq e^{3 B T}\left(f^{0}+\sum_{k=0}^{n} C_{k} \tau\right), \quad n=0,1, \cdots, N-1,
$$

where $\tau$ satisfies $B \tau<\frac{1}{2}$.

Theorem 4.1. Suppose $\kappa$ be piecewise smooth with discontinuity at certain cell-vertex $x_{j_{0}},(1 \leq$ $\left.j_{0} \leq J-1\right), u$ is the exact solution of (2.1a)-(2.1b), and $U^{n+1}$ is the solution of (2.7a)-(2.7b). Denote

$$
e_{j+\frac{1}{2}}^{n+1}=u\left(x_{j+\frac{1}{2}}, t^{n+1}\right)-U_{j+\frac{1}{2}}^{n+1}, \quad e_{h}^{k+1}=\left\{e_{j+\frac{1}{2}}^{k+1}\right\}_{j=-1}^{J},
$$

then we have the following estimation

$$
\left\|e_{h}^{n+1}\right\|_{d}+\left(\sum_{k=0}^{n} \tau\left\|\delta_{t} e_{h}^{k+1}\right\|_{d}^{2}\right)^{\frac{1}{2}} \leq C(h+\tau), \quad n=0,1, \cdots, N-1 .
$$

Proof. Denote

$$
u_{j+\frac{1}{2}}^{n+1}=u\left(x_{j+\frac{1}{2}}, t^{n+1}\right) .
$$

Substitute the exact solution $u$ into (2.7a), and we denote

$$
\begin{aligned}
L_{h} u_{j+\frac{1}{2}}^{n+1} & =\frac{u_{j+\frac{1}{2}}^{n+1}-u_{j+\frac{1}{2}}^{n}}{\tau}-\frac{1}{h_{j+\frac{1}{2}}}\left(\kappa_{j+1}^{n+1} \frac{u_{j+\frac{3}{2}}^{n+1}-u_{j+\frac{1}{2}}^{n+1}}{h_{j+1}}-\kappa_{j}^{n+1} \frac{u_{j+\frac{1}{2}}^{n+1}-u_{j-\frac{1}{2}}^{n+1}}{h_{j}}\right) \\
& =E_{1}+E_{2},
\end{aligned}
$$

where

$$
E_{1}=\frac{u_{j+\frac{1}{2}}^{n+1}-u_{j+\frac{1}{2}}^{n}}{\tau}, \quad E_{2}=-\frac{1}{h_{j+\frac{1}{2}}}\left(\kappa_{j+1}^{n+1} \frac{u_{j+\frac{3}{2}}^{n+1}-u_{j+\frac{1}{2}}^{n+1}}{h_{j+1}}-\kappa_{j}^{n+1} \frac{u_{j+\frac{1}{2}}^{n+1}-u_{j-\frac{1}{2}}^{n+1}}{h_{j}}\right) .
$$

Then we estimate $E_{1}$ and $E_{2}$, separately. 
Step 1. First, we consider $E_{1}$. From Taylor's expansion, we have

$$
\frac{u_{j+\frac{1}{2}}-u_{j+\frac{1}{2}}^{n}}{\tau}=u_{t}\left(x_{j+\frac{1}{2}}, t^{n+1}\right)+\tau r_{j}^{n+1}
$$

where $\left|r_{j}^{n+1}\right| \leq C$.

Step 2. Next, we consider $E_{2}$. From the Taylor's expansion, we have

$$
\begin{aligned}
& u_{j+\frac{1}{2}}^{n+1}=u_{j}^{n+1}+u_{x}^{+} \frac{h_{j+\frac{1}{2}}}{2}+\mathcal{O}\left(h^{2}\right), \\
& u_{j-\frac{1}{2}}^{n+1}=u_{j}^{n+1}-u_{x}^{-} \frac{h_{j-\frac{1}{2}}}{2}+\mathcal{O}\left(h^{2}\right),
\end{aligned}
$$

where

$$
u_{x}^{+}=u_{x}\left(x_{j}+0, t^{n+1}\right), \quad u_{x}^{-}=u_{x}\left(x_{j}-0, t^{n+1}\right) .
$$

Recall the definition of $\kappa_{j}^{n+1}$ that

$$
\kappa_{j}^{n+1}=\frac{2 h_{j}}{\frac{h_{j-\frac{1}{2}}}{\kappa_{j-\frac{1}{2}}^{n+1}}+\frac{h_{j+\frac{1}{2}}}{\kappa_{j+\frac{1}{2}}^{n+1}}} .
$$

Then from (4.3a), (4.3b) and (4.4), we have

$$
\kappa_{j}^{n+1} \frac{u_{j+\frac{1}{2}}^{n+1}-u_{j-\frac{1}{2}}^{n+1}}{h_{j}}=\frac{u_{x}^{+} h_{j+\frac{1}{2}}+u_{x}^{-} h_{j-\frac{1}{2}}}{\frac{h_{j-\frac{1}{2}}}{\kappa_{j-\frac{1}{2}}^{n+1}}+\frac{h_{j+\frac{1}{2}}}{\kappa_{j+\frac{1}{2}}^{n+1}}}+\mathcal{O}(h) .
$$

Denote $\mathcal{F}_{j}^{n+1}=F\left(x_{j}, t^{n+1}\right)$. It follows from the continuity of flux that $\mathcal{F}_{j}^{n+1}=$ $-\kappa^{+} u_{x}^{+}=-\kappa^{-} u_{x}^{-}$, where

$$
\kappa^{+}=\kappa\left(x_{j}+0, t^{n+1}\right), \quad \kappa^{-}=\kappa\left(x_{j}-0, t^{n+1}\right) .
$$

Then we have

$$
\kappa_{j}^{n+1} \frac{u_{j+\frac{1}{2}}^{n+1}-u_{j-\frac{1}{2}}^{n+1}}{h_{j}}=-\mathcal{F}_{j}^{n+1} \frac{\frac{h_{j+\frac{1}{2}}}{\kappa^{+}}+\frac{h_{j-\frac{1}{2}}}{\kappa^{-}}}{\frac{h_{j-\frac{1}{2}}}{\kappa_{j+\frac{1}{2}}^{n+1}}+\frac{1}{\kappa_{j-\frac{1}{2}}^{n+1}}}+\mathcal{O}(h) .
$$

Since $\kappa$ is piecewise smooth, thus we have

$$
\kappa^{+}=\kappa_{j-\frac{1}{2}}+\mathcal{O}(h), \quad \kappa^{-}=\kappa_{j-\frac{1}{2}}+\mathcal{O}(h),
$$


which implies

$$
\frac{\frac{h_{j+\frac{1}{2}}}{\kappa^{+}}+\frac{h_{j-\frac{1}{2}}}{\kappa^{-}}}{\frac{h_{j-\frac{1}{2}}}{\kappa_{j+\frac{1}{2}}}+\frac{1}{\kappa_{j-\frac{1}{2}}^{n+1}}}=1+\mathcal{O}(h) .
$$

Then we have

$$
\kappa_{j}^{n+1} \frac{u_{j+\frac{1}{2}}^{n+1}-u_{j-\frac{1}{2}}^{n+1}}{h_{j}}=-\mathcal{F}_{j}^{n+1}+R_{j}^{n+1},
$$

where $\left|R_{j}^{n+1}\right| \leq C h$.

From the Taylor expansion of $F_{x}$, we have

$$
\frac{1}{h_{j+\frac{1}{2}}}\left(\mathcal{F}_{j+1}^{n+1}-\mathcal{F}_{j}^{n+1}\right)=F_{x}\left(x_{j+\frac{1}{2}}, t^{n+1}\right)+\mathcal{O}\left(h^{2}\right) .
$$

Then we have

$$
E_{2}=F_{x}\left(x_{j+\frac{1}{2}}, t^{n+1}\right)-\frac{1}{h_{j+\frac{1}{2}}}\left(R_{j+1}^{n+1}-R_{j}^{n+1}\right)+\mathcal{O}\left(h^{2}\right) .
$$

Step 3. Combining (4.1), (4.2), and (4.5), we have

$$
\begin{aligned}
L_{h} u_{j+\frac{1}{2}}^{n+1} & =u_{t}-F_{x}+\tau r_{j+\frac{1}{2}}^{n+1}-\frac{1}{h_{j+\frac{1}{2}}}\left(R_{j+1}^{n+1}-R_{j}^{n+1}\right)+\mathcal{O}\left(h^{2}\right) \\
& =f_{j+\frac{1}{2}}^{n+1}+\tau r_{j+\frac{1}{2}}^{n+1}-\frac{1}{h_{j+\frac{1}{2}}}\left(R_{j+1}^{n+1}-R_{j}^{n+1}\right)+\mathcal{O}\left(h^{2}\right) .
\end{aligned}
$$

Step 4. Denote $e_{j+\frac{1}{2}}^{n+1}=u_{j+\frac{1}{2}}^{n+1}-U_{j+\frac{1}{2}}^{n+1}$. Since $L_{h} U_{j+\frac{1}{2}}^{n+1}=f_{j+\frac{1}{2}}^{n+1}$, then we have

$$
L_{h} e_{j+\frac{1}{2}}^{n+1}=\tau r_{j+\frac{1}{2}}^{n+1}-\frac{1}{h_{j+\frac{1}{2}}}\left(R_{j+1}^{n+1}-R_{j}^{n+1}\right)+\mathcal{O}\left(h^{2}\right) .
$$

Thus, $e_{j+\frac{1}{2}}^{n+1}$ satisfies the following equations

$$
\begin{array}{ll}
\frac{e_{j+\frac{1}{2}}^{n+1}-e_{j+\frac{1}{2}}^{n}}{\tau}-\frac{1}{h_{j+\frac{1}{2}}}\left(\kappa_{j+1}^{n+1} \frac{e_{j+\frac{3}{2}}^{n+1}-e_{j+\frac{1}{2}}^{n+1}}{h_{j+1}}-\kappa_{j}^{n+1} \frac{e_{j+\frac{1}{2}}^{n+1}-e_{j-\frac{1}{2}}^{n+1}}{h_{j}}\right) \\
=\tau r_{j+\frac{1}{2}}^{n+1}-\frac{1}{h_{j+\frac{1}{2}}}\left(R_{j+1}^{n+1}-R_{j}^{n+1}\right)+\mathcal{O}\left(h^{2}\right), & 0 \leq j \leq J-1, \quad 0 \leq n \leq N-1, \\
e_{-\frac{1}{2}}^{n+1}=e_{J+\frac{1}{2}}^{n+1}=0, & 0 \leq n \leq N-1, \\
e_{j+\frac{1}{2}}^{0}=0, & -1 \leq j \leq J .
\end{array}
$$


Multiplying (4.7) by $e_{j+\frac{1}{2}}^{n+1} h_{j+\frac{1}{2}}$, summing over $j=0,1, \cdots, J-1$, and using the discrete integration by parts, we have

$$
\begin{aligned}
& \frac{1}{2 \tau}\left(\left\|e_{h}^{n+1}\right\|_{d}^{2}-\left\|e_{h}^{n}\right\|_{d}^{2}+\left\|e_{h}^{n+1}-e_{h}^{n}\right\|_{d}^{2}\right)+\sum_{j=0}^{J-1} \kappa_{j}^{n+1}\left|\frac{e_{j+\frac{1}{2}}^{n+1}-e_{j-\frac{1}{2}}^{n+1}}{h_{j}}\right|^{2} h_{j} \\
= & \sum_{j=0}^{J} R_{j}^{n+1} \frac{e_{j+\frac{1}{2}}^{n+1}-e_{j-\frac{1}{2}}^{n+1}}{h_{j}} h_{j}+\tau \sum_{j=0}^{J-1} r_{j+\frac{1}{2}}^{n+1} e_{j+\frac{1}{2}}^{n+1} h_{j+\frac{1}{2}}+\sum_{j=0}^{J-1} \mathcal{O}\left(h^{2}\right) e_{j+\frac{1}{2}}^{n+1} h_{j+\frac{1}{2}} .
\end{aligned}
$$

Since $\left|R_{j}^{n+1}\right| \leq C h,\left|r_{j+\frac{1}{2}}^{n+1}\right| \leq C$, and $\kappa \geq \sigma_{0}$, using the Cauchy inequality $a b \leq \varepsilon a^{2}+\frac{1}{4 \varepsilon} b^{2}$, we have

$$
\begin{aligned}
& \frac{1}{2 \tau}\left(\left\|e_{h}^{n+1}\right\|_{d}^{2}-\left\|e_{h}^{n}\right\|_{d}^{2}+\left\|e_{h}^{n+1}-e_{h}^{n}\right\|_{d}^{2}\right)+\sum_{j=0}^{J-1} \kappa_{j}^{n+1}\left|\frac{e_{j+\frac{1}{2}}^{n+1}-e_{j-\frac{1}{2}}^{n+1}}{h_{j}}\right|^{2} h_{j} \\
\leq & C\left(\sum_{j=0}^{J} \kappa_{j}^{n+1}\left|\frac{e_{j+\frac{1}{2}}^{n+1}-e_{j-\frac{1}{2}}^{n+1}}{h_{j}}\right|^{2} h_{j}\right)^{\frac{1}{2}} h+C \tau\left\|e_{h}^{n+1}\right\|_{d}+\mathcal{O}\left(h^{2}\right)\left\|e_{h}^{n+1}\right\|_{d} \\
\leq & C h^{2}+\frac{1}{2} \sum_{j=0}^{J} \kappa_{j}^{n+1}\left|\frac{e_{j+\frac{1}{2}}^{n+1}-e_{j-\frac{1}{2}}^{n+1}}{h_{j}}\right|^{2} h_{j}+C \tau^{2}+\mathcal{O}\left(h^{4}\right)+\frac{1}{2}\left\|e_{h}^{n+1}\right\|_{d}^{2} .
\end{aligned}
$$

It follows that

$$
\frac{1}{2 \tau}\left(\left\|e_{h}^{n+1}\right\|_{d}^{2}-\left\|e_{h}^{n}\right\|_{d}^{2}\right)+\frac{1}{2} \sum_{j=0}^{J} \kappa_{j}^{n+1}\left|\frac{e_{j+\frac{1}{2}}^{n+1}-e_{j-\frac{1}{2}}^{n+1}}{h_{j}}\right|^{2} h_{j} \leq C h^{2}+C \tau^{2}+\frac{1}{2}\left\|e_{h}^{n+1}\right\|_{d}^{2} .
$$

Since $\kappa \geq \sigma_{0}$, multiplying (4.8) by $2 \tau$ leads to

$$
\left\|e_{h}^{n+1}\right\|_{d}^{2}-\left\|e_{h}^{n}\right\|_{d}^{2} \leq 2 \tau C\left(h^{2}+\tau^{2}\right)+\tau\left\|e_{h}^{n+1}\right\|_{d}^{2} .
$$

By using the Discrete Gronwall's inequality to (4.9) yields,

$$
\begin{aligned}
\left\|e_{h}^{n+1}\right\|_{d}^{2} & \leq e^{3 T}\left(\left\|e_{h}^{0}\right\|_{d}^{2}+\sum_{k=0}^{n} 2 \tau C\left(h^{2}+\tau^{2}\right)\right) \\
& \leq C\left(\tau^{2}+h^{2}\right) .
\end{aligned}
$$

Derivativing (4.9) about $t$ and summing over $k=0,1, \cdots, n+1$, and we have

$$
\left\|e_{h}^{n+1}\right\|_{d}^{2}+\sum_{k=0}^{n} \sigma_{0} \tau\left\|\delta_{t} e_{h}^{k+1}\right\|_{d}^{2} \leq C\left(h^{2}+\tau^{2}\right)+\sum_{k=0}^{n}\left\|e_{h}^{k+1}\right\|_{d}^{2} \tau .
$$


From (4.10), for any $k$ we have

$$
\left\|e_{h}^{k+1}\right\|_{d}^{2} \leq C\left(\tau^{2}+h^{2}\right) .
$$

Thus we have

$$
\left\|e_{h}^{n+1}\right\|_{d}^{2}+\sum_{k=0}^{n} \sigma_{0} \tau\left\|\delta_{t} e_{h}^{k+1}\right\|_{d}^{2} \leq C\left(h^{2}+\tau^{2}\right)
$$

which implies

$$
\left\|e_{h}^{n+1}\right\|_{d}+\left(\sum_{k=0}^{n} \tau\left\|\delta_{t} e_{h}^{k+1}\right\|_{d}^{2}\right)^{\frac{1}{2}} \leq C(h+\tau) .
$$

Thus the proof is completed.

Remark 4.1. The proof of Theorem 4.1 is to analyze the truncation error, and to use the energy estimate to get convergence of errors. The proof of Theorem 5.3 in [13] is similar.

\section{Comparison to the classical finite volume method}

In this section, we consider the truncation errors of the new conservative gradient discretization scheme and the classical finite volume method on uniform meshes. The truncation errors in time direction of two schemes are $\mathcal{O}(\tau)$. In space direction, the new scheme's truncation error is $\mathcal{O}\left(h^{2}\right)$, but the classical scheme's truncation error is $\mathcal{O}(1)$, which implies the new scheme has smaller truncation error.

As we mentioned in Remark 2.1, the main difference between the conservative gradient discretization scheme and classical finite volume scheme is that they use different meshes. The classical finite volume scheme's uniform meshes are

$$
0=\tilde{x}_{0}<\tilde{x}_{1}<\cdots<\tilde{x}_{J-1}<\tilde{x}_{J}=l,
$$

where $\tilde{x}_{j}$ denotes the vertex for $0 \leq j \leq J, \tilde{x}_{0}$ and $\tilde{x}_{J}$ are the left and right boundaries of $Q$, which causes different meshes. The meshes are shown in Fig. 2. $\tilde{x}_{j+\frac{1}{2}}$ represents the center of the cell $\left[\tilde{x}_{j}, \tilde{x}_{j+1}\right]$. $\tilde{h}$ denotes the mesh size of classical finite volume scheme. The classical finite volume scheme on uniform meshes [19] is as follows:

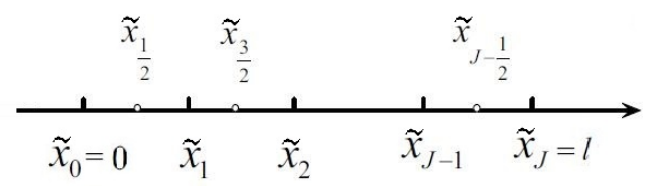

Figure 2: The meshes for classical finite volume method. 
Algorithm 5.1 (The classical finite volume scheme). For any $0 \leq j \leq J-1,0 \leq n \leq N-1$,

$$
\begin{aligned}
& \frac{\tilde{U}_{j+\frac{1}{2}}^{n+1}-\tilde{U}_{j+\frac{1}{2}}^{n}}{\tau}+\frac{1}{\tilde{h}}\left(\tilde{F}_{j+1}^{n+1}-\tilde{F}_{j}^{n+1}\right)=f_{j+\frac{1}{2}}^{n+1} \\
& \tilde{U}_{0}^{n+1}=\tilde{U}_{J}^{n+1}=0, \\
& \tilde{U}_{j+\frac{1}{2}}^{0}=u_{j+\frac{1}{2}}^{0} .
\end{aligned}
$$

The numerical flux is defined as follows

$$
\begin{aligned}
& \tilde{F}_{0}^{n+1}=-\kappa_{\frac{1}{2}}^{n+1} \frac{2\left(\tilde{U}_{\frac{1}{2}}^{n+1}-\tilde{U}_{0}^{n+1}\right)}{\tilde{h}}, \\
& \tilde{F}_{j}^{n+1}=-\kappa_{j}^{n+1} \frac{\tilde{U}_{j+\frac{1}{2}}^{n+1}-\tilde{U}_{j-\frac{1}{2}}^{n+1}, \quad 1 \leq j \leq J-1,}{\tilde{h}}, \\
& \tilde{F}_{J}^{n+1}=-\kappa_{J-\frac{1}{2}}^{n+1} \frac{2\left(\tilde{U}_{J}^{n+1}-\tilde{U}_{J-\frac{1}{2}}^{n+1}\right)}{\tilde{h}},
\end{aligned}
$$

where

$$
\kappa_{j}^{n+1}=\frac{2 \kappa_{j-\frac{1}{2}}^{n+1} \kappa_{j+\frac{1}{2}}^{n+1}}{\kappa_{j-\frac{1}{2}}^{n+1}+\kappa_{j+\frac{1}{2}}^{n+1}}, \quad f_{j+\frac{1}{2}}^{n+1}=\frac{1}{\tilde{h}} \int_{\tilde{x}_{j}}^{\tilde{x}_{j+1}} f\left(x, t^{n+1}\right) \mathrm{d} x, \quad u_{j+\frac{1}{2}}^{0}=\frac{1}{\tilde{h}} \int_{\tilde{x}_{j}}^{\tilde{x}_{j+1}} u^{0}(x) \mathrm{d} x .
$$

The different meshes cause the different discretization near the boundary cells. As we have described in Section 2, on the left boundary cell $\left(x_{0}, x_{1}\right)$, the conservative gradient discretization scheme (2.7a) is

$$
\frac{U_{\frac{1}{2}}^{n+1}-U_{\frac{1}{2}}^{n}}{\tau}-\frac{1}{h}\left(\kappa_{1}^{n+1} \frac{U_{\frac{3}{2}}^{n+1}-U_{\frac{1}{2}}^{n+1}}{h}-\kappa_{0}^{n+1} \frac{U_{\frac{1}{2}}^{n+1}-U_{-\frac{1}{2}}^{n+1}}{h}\right)=f_{\frac{1}{2}}^{n+1} .
$$

Next, we analyze the local truncation error of (5.4). Denote $u_{j+\frac{1}{2}}$ the value of exact solution at cell center $u\left(x_{j+\frac{1}{2}}, t^{n+1}\right)$ and $u_{j}$ the value of exact solution at vertex $u\left(x_{j}, t^{n+1}\right)$. Here we omit the subscript $n+1$. The Taylor expansion of $u_{\frac{1}{2}}$ and $u_{-\frac{1}{2}}$ at $x_{0}$ are

$$
\begin{aligned}
& u_{\frac{1}{2}}=u_{0}+\frac{h}{2} u_{0}^{\prime}+\frac{1}{2}\left(\frac{h}{2}\right)^{2} u_{0}^{\prime \prime}+\frac{1}{6}\left(\frac{h}{2}\right)^{3} u_{0}^{\prime \prime \prime}+\mathcal{O}\left(h^{4}\right), \\
& u_{-\frac{1}{2}}=u_{0}-\frac{h}{2} u_{0}^{\prime}+\frac{1}{2}\left(\frac{h}{2}\right)^{2} u_{0}^{\prime \prime}-\frac{1}{6}\left(\frac{h}{2}\right)^{3} u_{0}^{\prime \prime \prime}+\mathcal{O}\left(h^{4}\right) .
\end{aligned}
$$

Thus,

$$
\kappa_{0} \frac{u_{\frac{1}{2}}-u_{-\frac{1}{2}}}{h}=\kappa_{0}\left(u_{0}^{\prime}+\frac{1}{6}\left(\frac{h}{2}\right)^{2} u_{0}^{\prime \prime \prime}\right)+\mathcal{O}\left(h^{4}\right)
$$


Similarly, the Taylor expansions of $u_{\frac{1}{2}}$ and $u_{\frac{3}{2}}$ at $x_{1}$ lead to

$$
\kappa_{1} \frac{u_{\frac{3}{2}}-u_{\frac{1}{2}}}{h}=\kappa_{1}\left(u_{1}^{\prime}+\frac{1}{6}\left(\frac{h}{2}\right)^{2} u_{1}^{\prime \prime \prime}\right)+\mathcal{O}\left(h^{4}\right) .
$$

Notice that

$$
\begin{aligned}
& \kappa_{1} u_{1}^{\prime}-\kappa_{0} u_{0}^{\prime}=h\left(\kappa_{\frac{1}{2}} u_{\frac{1}{2}}^{\prime}\right)^{\prime}+\mathcal{O}\left(h^{3}\right), \\
& \kappa_{1} u_{1}^{\prime \prime \prime}-\kappa_{0} u_{0}^{\prime \prime \prime}=h\left(\kappa_{\frac{1}{2}} u_{\frac{1}{2}}^{\prime \prime \prime}\right)^{\prime}+\mathcal{O}\left(h^{3}\right),
\end{aligned}
$$

from (5.5)-(5.6) we have

$$
-\frac{1}{h}\left(\kappa_{1} \frac{u_{\frac{3}{2}}-u_{\frac{1}{2}}}{h}-\kappa_{0} \frac{u_{\frac{1}{2}}-u_{-\frac{1}{2}}}{h}\right)=-\left(\kappa_{\frac{1}{2}} u_{\frac{1}{2}}^{\prime}\right)^{\prime}+\mathcal{O}\left(h^{2}\right),
$$

which implies the local spatial truncation error of (5.4) is $\mathcal{O}\left(h^{2}\right)$.

For any $1 \leq j \leq J-1$, in the same way we can prove that the local spatial truncation error is $\mathcal{O}\left(h^{2}\right)$ at each $x_{j+\frac{1}{2}}$. Hence, the spatial truncation error is $\mathcal{O}\left(h^{2}\right)$.

For the classical finite volume method (5.2a)-(5.2c), on the left boundary cell $\left(\tilde{x}_{0}, \tilde{x}_{1}\right)$ the numerical scheme is

$$
\frac{\tilde{U}_{\frac{1}{2}}^{n+1}-\tilde{U}_{\frac{1}{2}}^{n}}{\tau}-\frac{1}{\tilde{h}}\left(\tilde{\kappa}_{1}^{n+1} \frac{\tilde{U}_{\frac{3}{2}}^{n+1}-\tilde{U}_{\frac{1}{2}}^{n+1}}{\tilde{h}}-\tilde{\kappa}_{0}^{n+1} \frac{\tilde{U}_{\frac{1}{2}}^{n+1}-\tilde{U}_{0}^{n+1}}{\frac{1}{2} \tilde{h}}\right)=f_{\frac{1}{2}}^{n+1} .
$$

The Taylor expansion for the term $\tilde{\kappa}_{1} \frac{\tilde{u}_{3}-\tilde{u}_{\frac{1}{2}}}{\hat{h}}$ is the same

$$
\tilde{\kappa}_{1} \frac{\tilde{u}_{\frac{3}{2}}-\tilde{u}_{\frac{1}{2}}}{\tilde{h}}=\tilde{\kappa}_{1}\left(\tilde{u}_{1}^{\prime}+\frac{1}{6}\left(\frac{\tilde{h}}{2}\right)^{2} \tilde{u}_{1}^{\prime \prime \prime}\right)+\mathcal{O}\left(\tilde{h}^{4}\right) .
$$

For the term $\tilde{\kappa}_{0} \frac{\tilde{u}_{\frac{1}{2}}-\tilde{u}_{0}}{\frac{1}{2} \tilde{h}}$, we have

$$
\tilde{\kappa}_{0} \frac{\tilde{u}_{\frac{1}{2}}-\tilde{u}_{0}}{\frac{1}{2} \tilde{h}}=\tilde{\kappa}_{0}\left(\tilde{u}_{0}^{\prime}+\frac{1}{2}\left(\frac{\tilde{h}}{2}\right) \tilde{u}_{0}^{\prime \prime}+\frac{1}{6}\left(\frac{\tilde{h}}{2}\right)^{2} \tilde{u}_{0}^{\prime \prime \prime}\right)+\mathcal{O}\left(\tilde{h}^{3}\right) .
$$

Notice that

$$
\begin{aligned}
& \tilde{\kappa}_{1} \tilde{u}_{1}^{\prime}-\tilde{\kappa}_{0} \tilde{u}_{0}^{\prime}=\tilde{h}\left(\tilde{\kappa}_{\frac{1}{2}} \tilde{u}_{\frac{1}{2}}^{\prime}\right)^{\prime}+\mathcal{O}\left(\tilde{h}^{3}\right), \\
& \tilde{\kappa}_{1} \tilde{u}_{1}^{\prime \prime \prime}-\tilde{\kappa}_{0} \tilde{u}_{0}^{\prime \prime \prime}=\tilde{h}\left(\tilde{\kappa}_{\frac{1}{2}} \tilde{u}_{\frac{1}{2}}^{\prime \prime \prime}\right)^{\prime}+\mathcal{O}\left(\tilde{h}^{3}\right),
\end{aligned}
$$


from (5.8)-(5.9) we have

$$
-\frac{1}{\tilde{h}}\left(\tilde{\kappa}_{1} \frac{\tilde{u}_{\frac{3}{2}}-\tilde{u}_{\frac{1}{2}}}{\tilde{h}}-\tilde{\kappa}_{0} \frac{\tilde{u}_{\frac{1}{2}}-\tilde{u}_{0}}{\frac{1}{2} \tilde{h}}\right)=-\left(\tilde{\mathcal{K}}_{\frac{1}{2}} \tilde{u}_{\frac{1}{2}}^{\prime}\right)^{\prime}-\frac{1}{4} \tilde{\mathcal{K}}_{0} \tilde{u}_{0}^{\prime \prime}+\mathcal{O}\left(\tilde{h}^{2}\right) .
$$

Since the term $-\frac{1}{4} \tilde{\kappa}_{0} \tilde{u}_{0}^{\prime \prime}$ is $\mathcal{O}(1)$, the local spatial truncation error of $(5.7)$ is $\mathcal{O}(1)$. Hence, the spatial truncation error is $\mathcal{O}(1)$.

Remark 5.1. It should be noticed that whatever the boundary conditions $\tilde{U}_{0}^{n+1}$ and $\tilde{U}_{J}^{n+1}$ are, (5.10) always holds. For any Dirichlet boundary condition, the term $-\frac{1}{4} \tilde{\kappa}_{0} \tilde{u}_{0}^{\prime \prime}$ does not vanish in general, and then the spatial truncation error of the classical finite volume scheme is $\mathcal{O}(1)$.

\section{Numerical experiments}

In this section, we compare the errors between the new conservative gradient discretization scheme (2.7a)-(2.7b) and the classical finite volume scheme. The results of experiments show that the new scheme is better than the classical scheme in terms of flux error, $L^{2}$ error and $L^{\infty}$ error.

Define the $L^{2}$ error of solution by

$$
e_{2}^{u}=\left(\sum_{i=0}^{J-1}\left(u\left(x_{i+\frac{1}{2}}, T\right)-U_{i+\frac{1}{2}}^{N}\right)^{2} h_{i+\frac{1}{2}}\right)^{\frac{1}{2}},
$$

and the $L^{\infty}$ error of solution by

$$
e_{\infty}^{u}=\max _{-1 \leq i \leq J}\left\{\left|u\left(x_{i+\frac{1}{2}}, T\right)-U_{i+\frac{1}{2}}^{N}\right|\right\},
$$

and the error of flux by

$$
e_{2}^{F}=\left(\sum_{i=0}^{J}\left(F\left(x_{i}, T\right)-F_{i}^{N}\right)^{2} h_{i}\right)^{\frac{1}{2}}
$$

Define the conservative error by

$$
e_{d c o n}=\left|\sum_{j=0}^{J-1} U_{j+\frac{1}{2}}^{N} h_{j+\frac{1}{2}}-\sum_{j=0}^{J-1} U_{j+\frac{1}{2}}^{0} h_{j+\frac{1}{2}}-\sum_{n=1}^{N}\left(F_{J-1}^{n}-F_{0}^{n}\right) \tau-\sum_{n=1}^{N} \sum_{j=0}^{J-1} f_{j+\frac{1}{2}}^{n} h_{j+\frac{1}{2}} \tau\right|,
$$

and the total energy error by

$$
e_{\text {energy }}=\left|\int_{0}^{l} u(x, T) \mathrm{d} x-\sum_{j=-1}^{J} U_{j+\frac{1}{2}}^{N} h_{j+\frac{1}{2}}\right| .
$$


We verify the conservation of the scheme $(2.7 \mathrm{a})-(2.7 \mathrm{~b})$ by testing on the exact solution $u=e^{-\pi^{2} t} \cos (\pi x)$ on $(0,1)$ with $\kappa=1$. In this case the source term $f=0$ and the flux at $x=0$ and $x=1$ are zero. The result shows that the conservative error $e_{d c o n}$ is of scale $10^{-14}$, which is caused by rounding error.

In the numerical experiments, we test the new conservative gradient discretization scheme (2.7a)-(2.7b) and classical finite volume scheme on random meshes. We take the domain $Q=(0,1)$ in the following numerical experiments. We first generate the uniform meshes with mesh size $h$, then disturb the vertex by random numbers lying in $\left(-\frac{\sigma h}{2}, \frac{\sigma h}{2}\right)$, where $\sigma=0.5$. Especially, the final random number is the same as the first random number.

\subsection{Example 1}

In the first example, we test the numerical schemes for inhomogeneous coefficient $\kappa$. We take $u(x, t)=e^{-t} \cos (\pi x), \kappa=\sin (\pi x)+2$, and $T=1$. In order to show the spatial convergence order, the time step needs to be sufficiently small, hence we take $\tau=5 \mathrm{E}-6$. We test the new scheme and classical scheme on meshes of sizes $1 / 10,1 / 20, \cdots, 1 / 320$, separately. The results are shown in Tables 1-2.

From Tables 1-2 we see that all the flux error, $L^{2}$ error and $L^{\infty}$ error are of second order convergence for both two schemes. Compared to the classical finite volume scheme, we can see that for the new scheme, the flux error is reduced by $40 \%$, the $L^{2}$ error is reduced by about $15 \%$, and the $L^{\infty}$ error is reduced by about $13 \%$.

We take the sufficiently fine meshes to obtain the temporal convergence order of the new scheme, where $h=1 \mathrm{E}-5$. Then we take time steps $1 / 10,1 / 20, \cdots, 1 / 320$, separately.

Table 1: The errors and spatial convergence orders of conservative gradient discretization scheme.

\begin{tabular}{||ccccccccc||}
\hline$h$ & $e_{2}^{F}$ & order & $e_{2}^{u}$ & order & $e_{\infty}^{u}$ & order & $e_{\text {energy }}$ & order \\
\hline $1 / 10$ & $1.44 \mathrm{E}-02$ & & $3.50 \mathrm{E}-03$ & & $5.84 \mathrm{E}-03$ & & $2.65 \mathrm{E}-03$ & \\
$1 / 20$ & $3.19 \mathrm{E}-03$ & 2.02 & $8.40 \mathrm{E}-04$ & 1.91 & $1.50 \mathrm{E}-03$ & 1.82 & $6.81 \mathrm{E}-04$ & 1.82 \\
$1 / 40$ & $8.15 \mathrm{E}-04$ & 1.90 & $2.12 \mathrm{E}-04$ & 1.92 & $4.52 \mathrm{E}-04$ & 1.67 & $1.70 \mathrm{E}-04$ & 1.93 \\
$1 / 80$ & $2.03 \mathrm{E}-04$ & 1.97 & $5.26 \mathrm{E}-05$ & 1.97 & $1.06 \mathrm{E}-04$ & 2.06 & $4.40 \mathrm{E}-05$ & 1.91 \\
$1 / 160$ & $5.13 \mathrm{E}-05$ & 1.96 & $1.38 \mathrm{E}-05$ & 1.91 & $2.99 \mathrm{E}-05$ & 1.81 & $1.18 \mathrm{E}-05$ & 1.88 \\
$1 / 320$ & $1.27 \mathrm{E}-05$ & 2.00 & $3.31 \mathrm{E}-06$ & 2.05 & $7.62 \mathrm{E}-06$ & 1.96 & $2.84 \mathrm{E}-06$ & 2.05 \\
\hline
\end{tabular}

Table 2: The errors and spatial convergence orders of classical finite volume scheme.

\begin{tabular}{||ccccccccc||}
\hline$h$ & $e_{2}^{F}$ & order & $e_{2}^{u}$ & order & $e_{\infty}^{u}$ & order & $e_{\text {energy }}$ & order \\
\hline $1 / 10$ & $2.35 \mathrm{E}-02$ & & $4.03 \mathrm{E}-03$ & & $6.10 \mathrm{E}-03$ & & $4.35 \mathrm{E}-04$ & \\
$1 / 20$ & $5.79 \mathrm{E}-03$ & 1.87 & $9.91 \mathrm{E}-04$ & 1.88 & $1.91 \mathrm{E}-03$ & 1.55 & $4.48 \mathrm{E}-05$ & 3.04 \\
$1 / 40$ & $1.41 \mathrm{E}-03$ & 1.97 & $2.55 \mathrm{E}-04$ & 1.89 & $5.36 \mathrm{E}-04$ & 1.77 & $1.61 \mathrm{E}-05$ & 1.43 \\
$1 / 80$ & $3.47 \mathrm{E}-04$ & 1.99 & $6.28 \mathrm{E}-05$ & 1.98 & $1.37 \mathrm{E}-04$ & 1.93 & $1.97 \mathrm{E}-06$ & 2.97 \\
$1 / 160$ & $8.76 \mathrm{E}-05$ & 1.97 & $1.56 \mathrm{E}-05$ & 1.99 & $3.58 \mathrm{E}-05$ & 1.92 & $2.06 \mathrm{E}-07$ & 3.23 \\
$1 / 320$ & $2.17 \mathrm{E}-05$ & 2.01 & $3.83 \mathrm{E}-06$ & 2.02 & $8.72 \mathrm{E}-06$ & 2.03 & $7.12 \mathrm{E}-08$ & 1.52 \\
\hline
\end{tabular}


Table 3: The errors and temporal convergence orders of conservative gradient discretization scheme.

\begin{tabular}{||ccccccccc||}
\hline$\tau$ & $e_{2}^{F}$ & order & $e_{2}^{u}$ & order & $e_{\infty}^{u}$ & order & $e_{\text {energy }}$ & order \\
\hline $1 / 10$ & $1.90 \mathrm{E}-03$ & & $1.18 \mathrm{E}-04$ & & $1.64 \mathrm{E}-04$ & & $2.98 \mathrm{E}-09$ & \\
$1 / 20$ & $9.33 \mathrm{E}-04$ & 1.02 & $5.78 \mathrm{E}-05$ & 1.02 & $8.06 \mathrm{E}-05$ & 1.02 & $2.98 \mathrm{E}-09$ & 0.00 \\
$1 / 40$ & $4.62 \mathrm{E}-04$ & 1.01 & $2.86 \mathrm{E}-05$ & 1.01 & $4.00 \mathrm{E}-05$ & 1.01 & $2.97 \mathrm{E}-09$ & 0.00 \\
$1 / 80$ & $2.30 \mathrm{E}-04$ & 1.01 & $1.43 \mathrm{E}-05$ & 1.01 & $1.99 \mathrm{E}-05$ & 1.01 & $2.97 \mathrm{E}-09$ & 0.00 \\
$1 / 160$ & $1.15 \mathrm{E}-04$ & 1.00 & $7.11 \mathrm{E}-06$ & 1.00 & $9.93 \mathrm{E}-06$ & 1.00 & $2.97 \mathrm{E}-09$ & 0.00 \\
$1 / 320$ & $5.74 \mathrm{E}-05$ & 1.00 & $3.55 \mathrm{E}-06$ & 1.00 & $4.96 \mathrm{E}-06$ & 1.00 & $2.97 \mathrm{E}-09$ & 0.00 \\
\hline
\end{tabular}

The results are shown in Table 3. The numerical results show that the flux error, $L^{2}$ error and $L^{\infty}$ error achieve first order convergence in time direction.

\subsection{Example 2}

In the second example, we test the numerical schemes for discontinuous coefficient. We take

$$
u=\left\{\begin{array}{ll}
e^{-t} \cos (\pi x), & x \leq 1 / 2, \\
2 e^{-t} \cos (\pi x), & x>1 / 2,
\end{array} \text { and } \quad \kappa= \begin{cases}2, & x \leq 1 / 2 \\
1, & x>1 / 2 .\end{cases}\right.
$$

$T$ is set to be 1 and the time step is $\tau=5 \mathrm{E}-6$. We test the new scheme and classical scheme on meshes of sizes $1 / 10,1 / 20, \cdots, 1 / 320$, separately. The results are shown in Tables $4-5$.

Also, the optimal convergence orders are observed. Compared to the classical finite volume scheme, from Tables $4-5$ we see that for the new scheme, the flux error is reduced by $70 \%$, the $L^{2}$ error is reduced by about $10 \%$, and the $L^{\infty}$ error is reduced by about $20 \%$.

Table 4: The errors and spatial convergence orders of conservative gradient discretization scheme.

\begin{tabular}{||ccccccccc||}
\hline$h$ & $e_{2}^{F}$ & order & $e_{2}^{u}$ & order & $e_{\infty}^{u}$ & order & $e_{\text {energy }}$ & order \\
\hline $1 / 10$ & $3.70 \mathrm{E}-03$ & & $4.06 \mathrm{E}-03$ & & $7.23 \mathrm{E}-03$ & & $3.74 \mathrm{E}-03$ & \\
$1 / 20$ & $9.89 \mathrm{E}-04$ & 1.90 & $1.14 \mathrm{E}-03$ & 1.84 & $2.31 \mathrm{E}-03$ & 1.65 & $1.07 \mathrm{E}-03$ & 1.81 \\
$1 / 40$ & $2.62 \mathrm{E}-04$ & 1.92 & $3.46 \mathrm{E}-04$ & 1.72 & $8.07 \mathrm{E}-04$ & 1.52 & $3.12 \mathrm{E}-04$ & 1.78 \\
$1 / 80$ & $6.74 \mathrm{E}-05$ & 1.96 & $8.02 \mathrm{E}-05$ & 2.11 & $1.92 \mathrm{E}-04$ & 2.07 & $7.59 \mathrm{E}-05$ & 2.04 \\
$1 / 160$ & $1.70 \mathrm{E}-05$ & 1.99 & $2.14 \mathrm{E}-05$ & 1.91 & $5.65 \mathrm{E}-05$ & 1.77 & $2.02 \mathrm{E}-05$ & 1.91 \\
$1 / 320$ & $4.39 \mathrm{E}-06$ & 1.95 & $5.19 \mathrm{E}-06$ & 2.04 & $1.37 \mathrm{E}-05$ & 2.04 & $4.95 \mathrm{E}-06$ & 2.03 \\
\hline
\end{tabular}

Table 5: The errors and spatial convergence orders of classical finite volume scheme.

\begin{tabular}{||ccccccccc||}
\hline$h$ & $e_{2}^{F}$ & order & $e_{2}^{u}$ & order & $e_{\infty}^{u}$ & order & $e_{\text {energy }}$ & order \\
\hline $1 / 10$ & $1.44 \mathrm{E}-02$ & & $5.72 \mathrm{E}-03$ & & $1.02 \mathrm{E}-02$ & & $1.84 \mathrm{E}-03$ & \\
$1 / 20$ & $3.74 \mathrm{E}-03$ & 1.80 & $1.43 \mathrm{E}-03$ & 1.85 & $3.18 \mathrm{E}-03$ & 1.56 & $4.90 \mathrm{E}-04$ & 1.77 \\
$1 / 40$ & $9.02 \mathrm{E}-04$ & 1.98 & $3.75 \mathrm{E}-04$ & 1.87 & $9.68 \mathrm{E}-04$ & 1.66 & $1.22 \mathrm{E}-04$ & 1.94 \\
$1 / 80$ & $2.20 \mathrm{E}-04$ & 2.00 & $9.35 \mathrm{E}-05$ & 1.97 & $2.46 \mathrm{E}-04$ & 1.94 & $3.20 \mathrm{E}-05$ & 1.89 \\
$1 / 160$ & $5.66 \mathrm{E}-05$ & 1.94 & $2.34 \mathrm{E}-05$ & 1.98 & $7.07 \mathrm{E}-05$ & 1.78 & $8.72 \mathrm{E}-06$ & 1.86 \\
$1 / 320$ & $1.39 \mathrm{E}-05$ & 2.02 & $5.75 \mathrm{E}-06$ & 2.02 & $1.68 \mathrm{E}-05$ & 2.06 & $2.05 \mathrm{E}-06$ & 2.08 \\
\hline
\end{tabular}




\subsection{Example 3}

In the third example, we test the numerical schemes for quasilinear equations. We still take $u(x, t)=e^{-t} \cos (\pi x)$, and we select $\kappa=u^{2}+5$. $T$ is set to be 1 and the time step is $\tau=5 \mathrm{E}-6$. We test the new scheme and classical scheme on meshes of sizes $1 / 10,1 / 20, \cdots$, 1/320, separately. The results are shown in Tables 6-7.

Table 6: The errors and spatial convergence orders of conservative gradient discretization scheme.

\begin{tabular}{||ccccccccc||}
\hline$h$ & $e_{2}^{F}$ & order & $e_{2}^{u}$ & order & $e_{\infty}^{u}$ & order & $e_{\text {energy }}$ & order \\
\hline $1 / 10$ & $1.76 \mathrm{E}-02$ & & $3.24 \mathrm{E}-03$ & & $5.17 \mathrm{E}-03$ & & $2.64 \mathrm{E}-03$ & \\
$1 / 20$ & $4.28 \mathrm{E}-03$ & 1.89 & $8.04 \mathrm{E}-04$ & 1.87 & $1.40 \mathrm{E}-03$ & 1.75 & $6.80 \mathrm{E}-04$ & 1.82 \\
$1 / 40$ & $1.14 \mathrm{E}-03$ & 1.84 & $2.05 \mathrm{E}-04$ & 1.90 & $4.17 \mathrm{E}-04$ & 1.68 & $1.73 \mathrm{E}-04$ & 1.90 \\
$1 / 80$ & $2.90 \mathrm{E}-04$ & 1.94 & $5.07 \mathrm{E}-05$ & 1.98 & $9.98 \mathrm{E}-05$ & 2.03 & $4.42 \mathrm{E}-05$ & 1.94 \\
$1 / 160$ & $7.19 \mathrm{E}-05$ & 2.00 & $1.32 \mathrm{E}-05$ & 1.93 & $2.88 \mathrm{E}-05$ & 1.78 & $1.16 \mathrm{E}-05$ & 1.91 \\
$1 / 320$ & $1.82 \mathrm{E}-05$ & 1.97 & $3.19 \mathrm{E}-06$ & 2.03 & $6.98 \mathrm{E}-06$ & 2.03 & $2.84 \mathrm{E}-06$ & 2.02 \\
\hline
\end{tabular}

Table 7: The errors and spatial convergence orders of classical finite volume scheme.

\begin{tabular}{||ccccccccc||}
\hline$h$ & $e_{2}^{F}$ & order & $e_{2}^{u}$ & order & $e_{\infty}^{u}$ & order & $e_{\text {energy }}$ & order \\
$1 / 10$ & $3.66 \mathrm{E}-02$ & & $3.66 \mathrm{E}-03$ & & $5.89 \mathrm{E}-03$ & & $3.12 \mathrm{E}-04$ & \\
$1 / 20$ & $9.28 \mathrm{E}-03$ & 1.83 & $9.24 \mathrm{E}-04$ & 1.84 & $1.79 \mathrm{E}-03$ & 1.59 & $4.34 \mathrm{E}-05$ & 2.64 \\
$1 / 40$ & $2.25 \mathrm{E}-03$ & 1.97 & $2.38 \mathrm{E}-04$ & 1.89 & $5.17 \mathrm{E}-04$ & 1.73 & $1.14 \mathrm{E}-05$ & 1.86 \\
$1 / 80$ & $5.54 \mathrm{E}-04$ & 1.98 & $5.88 \mathrm{E}-05$ & 1.98 & $1.35 \mathrm{E}-04$ & 1.91 & $1.56 \mathrm{E}-06$ & 2.81 \\
$1 / 160$ & $1.39 \mathrm{E}-04$ & 1.97 & $1.46 \mathrm{E}-05$ & 2.00 & $3.52 \mathrm{E}-05$ & 1.92 & $7.47 \mathrm{E}-08$ & 4.35 \\
$1 / 320$ & $3.45 \mathrm{E}-05$ & 2.01 & $3.57 \mathrm{E}-06$ & 2.02 & $8.61 \mathrm{E}-06$ & 2.02 & $4.68 \mathrm{E}-08$ & 0.67 \\
\hline
\end{tabular}

Also, the optimal convergence orders are observed. Compared to the classical finite volume scheme, from Tables 6-7 we see that for the new scheme, the flux error is reduced by $50 \%$, the $L^{2}$ error is reduced by about $12 \%$, and the $L^{\infty}$ error is reduced by about $20 \%$.

\subsection{Example 4}

In the last example, we test the numerical schemes for nonlinear equations. We still take $u(x, t)=e^{-t} \cos (\pi x)$, and we select $\kappa=u_{x}^{2}+10 . T$ is set to be 1 and the time step is $\tau=5 \mathrm{E}-6$. We test the new scheme and classical scheme on meshes of sizes $1 / 10,1 / 20, \cdots, 1 / 320$, separately. The results are shown in Tables 8-9.

Also, the optimal convergence orders are observed. Compared to the classical finite volume scheme, from Tables 8-9 we see that for the new scheme, the flux error is reduced by $50 \%$, the $L^{2}$ error is reduced by about $12 \%$, and the $L^{\infty}$ error is reduced by about $20 \%$.

\section{Conclusions}

We present a new conservative gradient discretization scheme for the parabolic equations. The new scheme is locally conservative on the new cell-centered meshes. Under 
Table 8: The errors and spatial convergence orders of conservative gradient discretization scheme.

\begin{tabular}{||ccccccccc||}
\hline$h$ & $e_{2}^{F}$ & order & $e_{2}^{u}$ & order & $e_{\infty}^{u}$ & order & $e_{\text {energy }}$ & order \\
\hline $1 / 10$ & $4.54 \mathrm{E}-02$ & & $3.22 \mathrm{E}-03$ & & $5.15 \mathrm{E}-03$ & & $2.51 \mathrm{E}-03$ & \\
$1 / 20$ & $1.01 \mathrm{E}-02$ & 2.01 & $7.94 \mathrm{E}-04$ & 1.87 & $1.37 \mathrm{E}-03$ & 1.77 & $6.60 \mathrm{E}-04$ & 1.79 \\
$1 / 40$ & $2.67 \mathrm{E}-03$ & 1.85 & $2.02 \mathrm{E}-04$ & 1.90 & $4.21 \mathrm{E}-04$ & 1.64 & $1.66 \mathrm{E}-04$ & 1.91 \\
$1 / 80$ & $6.76 \mathrm{E}-04$ & 1.94 & $5.02 \mathrm{E}-05$ & 1.97 & $1.00 \mathrm{E}-04$ & 2.03 & $4.30 \mathrm{E}-05$ & 1.92 \\
$1 / 160$ & $1.69 \mathrm{E}-04$ & 1.98 & $1.31 \mathrm{E}-05$ & 1.92 & $2.89 \mathrm{E}-05$ & 1.78 & $1.14 \mathrm{E}-05$ & 1.90 \\
$1 / 320$ & $4.26 \mathrm{E}-05$ & 1.98 & $3.16 \mathrm{E}-06$ & 2.05 & $7.08 \mathrm{E}-06$ & 2.02 & $2.76 \mathrm{E}-06$ & 2.04 \\
\hline
\end{tabular}

Table 9: The errors and spatial convergence orders of classical finite volume scheme.

\begin{tabular}{||ccccccccc||}
\hline$h$ & $e_{2}^{F}$ & order & $e_{2}^{u}$ & order & $e_{\infty}^{u}$ & order & $e_{\text {energy }}$ & order \\
\hline $1 / 10$ & $9.23 \mathrm{E}-02$ & & $3.67 \mathrm{E}-03$ & & $5.81 \mathrm{E}-03$ & & $4.33 \mathrm{E}-04$ & \\
$1 / 20$ & $2.30 \mathrm{E}-02$ & 1.86 & $9.12 \mathrm{E}-04$ & 1.86 & $1.78 \mathrm{E}-03$ & 1.59 & $4.24 \mathrm{E}-05$ & 3.11 \\
$1 / 40$ & $5.58 \mathrm{E}-03$ & 1.97 & $2.36 \mathrm{E}-04$ & 1.88 & $5.13 \mathrm{E}-04$ & 1.73 & $1.38 \mathrm{E}-05$ & 1.56 \\
$1 / 80$ & $1.37 \mathrm{E}-03$ & 1.98 & $5.82 \mathrm{E}-05$ & 1.98 & $1.34 \mathrm{E}-04$ & 1.91 & $1.68 \mathrm{E}-06$ & 2.99 \\
$1 / 160$ & $3.47 \mathrm{E}-04$ & 1.97 & $1.45 \mathrm{E}-05$ & 1.99 & $3.50 \mathrm{E}-05$ & 1.92 & $1.43 \mathrm{E}-07$ & 3.52 \\
$1 / 320$ & $8.57 \mathrm{E}-05$ & 2.01 & $3.54 \mathrm{E}-06$ & 2.02 & $8.56 \mathrm{E}-06$ & 2.02 & $6.53 \mathrm{E}-08$ & 1.12 \\
\hline
\end{tabular}

the framework of the gradient discretization method, we prove the new scheme is stable. We also prove that the scheme has $\mathcal{O}(\tau+h)$ convergence order for the linear problem with continuous and discontinuous coefficients, and it is convergent for the quasilinear problem with continuous coefficient on random meshes.

We compare the truncation errors of the new scheme $(2.7 \mathrm{a})-(2.7 \mathrm{~b})$ and the classical finite volume scheme (5.2a)-(5.2c) on uniform meshes with continuous coefficient. In this case, the truncation error of the new scheme is $\mathcal{O}\left(\tau+h^{2}\right)$, which is smaller than $\mathcal{O}(1)$ of the classical scheme. However, when the meshes are non-uniform or the coefficient is discontinuous, the truncation error of the new scheme $(2.7 \mathrm{a})-(2.7 \mathrm{~b})$ is $\mathcal{O}(1)$, and the theoretical convergence rate is $\mathcal{O}(\tau+h)$.

In numerical aspects, we compare the errors of two schemes with linear, quasilinear, and nonlinear diffusion coefficients. We observe that both schemes are of second order. Although the total energy error of the new scheme is larger than the classical scheme, the new scheme's flux error is reduced by $50 \%$, and the $L^{2}$ error is reduced by $12 \%$ and $L^{\infty}$ error is reduced by $20 \%$ on average, respectively. The results show that the new scheme has better numerical performance.

\section{Acknowledgements}

This work is partially supported by the National Natural Science Foundation of China (No. 11971069), NSAF (No. U1630249) and Science Challenge Project (No. TZ2016002). 


\section{References}

[1] I. A. Avatsmark, T. BARKVe, Ø. BøE AND T. MANNSETH, Discretization on non-orthogonal, quadrilateral grids for inhomogeneous, anisotropic media, J. Comput. Phys., 127 (1996), pp. 2-14.

[2] J. BENITO, F. UREÑA AND L. GAVETE, Solving parabolic and hyperbolic equations by the generalized finite difference method, J. Comput. Appl. Math., 209 (2007), pp. 208-233.

[3] F. BREZZI, A. BufFA AND K. LIPNIKOV, Mimetic finite differences for elliptic problems, ESAIM Math. Model. Numer. Anal., 43 (2009), pp. 277-295.

[4] P. CAstillo AND S. GóMEZ, Conservative local discontinuous Galerkin method for the fractional Klein-Gordon-Schrödinger system with generalized Yukawa interaction, Numer. Algorithms, 84 (2020), pp. 407-425.

[5] J. CHAN, On discretely entropy conservative and entropy stable discontinuous Galerkin methods, J. Comput. Phys., 362 (2018), pp. 346-374.

[6] K. CHRYSAFINOS AND N. J. WALKINGTON, Error estimates for the discontinuous Galerkin methods for parabolic equations, SIAM J. Numer. Anal., 44 (2006), pp. 349-366.

[7] Y. COUdière AND F. HubERT, A 3D discrete duality finite volume method for nonlinear elliptic equations, SIAM J. Sci. Comput., (2011).

[8] Y. Coudière, F. Hubert and G. MANZini, A CeVeFE DDFV Scheme for Discontinuous Anisotropic Permeability Tensors, Springer Proc. Math., (2011).

[9] W.-S. DON AND R. BORGES, Accuracy of the weighted essentially non-oscillatory conservative finite difference schemes, J. Comput. Phys., 250 (2013), pp. 347-372.

[10] J. DOUGLAS AND C.-S. HuANG, A locally conservative Eulerian-Lagrangian finite difference method for a parabolic equation, BIT Numer. Math., 41 (2001), pp. 480-489.

[11] O. DRblíKOVÁ, A. HANDlovičovÁ AND K. MiKUla, Error estimates of the finite volume scheme for the nonlinear tensor-driven anisotropic diffusion, Appl. Numer. Math., 59 (2009), pp. 2548-2570.

[12] O. DRblíKOVÁ AND K. Mikula, Convergence analysis of finite volume scheme for nonlinear tensor anisotropic diffusion in image processing, SIAM J. Numer. Anal., 46 (2008), pp. 37-60.

[13] J. Droniou, R. Eymard, T. Gallouët, C. Guichard And R. HERbin, The Gradient Discretisation Method, vol. 82 of Mathématiques et Applications, Springer International Publishing, Cham, 2018.

[14] J. Droniou, R. Eymard, T. Gallouet And R. Herbin, A unified approach to mimetic finite difference, hybrid finite volume and mixed finite volume methods, Math. Model. Methods Appl. Sci., 20 (2010), pp. 265-295.

[15] J. DRONIOU, R. EYMARD, T. GALLOUET AND R. HERBIN, Gradient schemes: a generic framework for the discretisation of linear, nonlinear and nonlocal elliptic and parabolic equations, Math. Model. Methods Appl. Sci., 23 (2013), pp. 2395-2432.

[16] J. DRONIOU, R. EYMARD AND R. HERBIN, Gradient schemes: generic tools for the numerical analysis of diffusion equations, ESAIM Math. Model. Numer. Anal., 50 (2016), pp. 749-781.

[17] K. ERIKSSON AND C. JOHNSON, Adaptive finite element methods for parabolic problems I: A linear model problem, SIAM J. Numer. Anal., 28 (1991), pp. 43-77.

[18] S. ERIKSSON, Q. ABbAS AND J. NORDSTRÖM, A stable and conservative method for locally adapting the design order of finite difference schemes, J. Comput. Phys., 230 (2011), pp. 42164231.

[19] R. Eymard, T. Gallouët AND R. Herbin, Finite volume Methods, Handb. Numer. Anal., Elsevier B. V., Amsterdam, (2000), pp. 713-1018.

[20] R. Eymard, C. Guichard AND R. Herbin, Benchmark 3D: the VAG Scheme, (2011), pp. 
1013-1022.

[21] R. EYMARD, C. Guichard AND R. Herbin, Small-stencil 3D schemes for diffusive flows in porous media, ESAIM Math. Model. Numer. Anal., 46 (2012), pp. 265-290.

[22] R. Eymard, A. Handlovicova, H. Raphaele, M. Karol and S. Olga, Applications of approximate gradient schemes for nonlinear parabolic equations, Appl. Math., 60 (2015), pp. 135-156.

[23] W. FenG, Z. GuO, J. S. Lowengrub AND S. M. WiSe, A mass-conservative adaptive FAS multigrid solver for cell-centered finite difference methods on block-structured, locally-cartesian grids, J. Comput. Phys., 352 (2018), pp. 463-497.

[24] B. Hou, D. LIANG AND H. ZHU, The conservative time high-order AVF compact finite difference schemes for two-dimensional variable coefficient acoustic wave equations, J. Sci. Comput., 80 (2019), pp. 1279-1309.

[25] S. KIM AND H. LIM, High-order schemes for acoustic waveform simulation, Appl. Numer. Math., 57 (2007), pp. 402-414.

[26] D. LING, J. CHENG AND C. W. SHU, Conservative high order positivity-preserving discontinuous Galerkin methods for linear hyperbolic and radiative transfer equations, J. Sci. Comput., 77 (2018), pp. 1801-1831.

[27] K. LipNiKov AND N. MoRgAN, A high-order conservative remap for discontinuous Galerkin schemes on curvilinear polygonal meshes, J. Comput. Phys., 399 (2019), 108931.

[28] K. LIPNIKOV, D. SVYATSKIY AND Y. VASSILEVSKI, Minimal stencil finite volume scheme with the discrete maximum principle, Russ. J. Numer. Anal. Math. Model., 27 (2012), pp. 369-385.

[29] B. RIVIÈRE, Discontinuous Galerkin methods for solving elliptic and parabolic equations, Society for Industrial and Applied Mathematics, Philadelphia, (2008).

[30] K. SHAHBAZI, High-order finite difference scheme for compressible multi-component flow computations, Comput. Fluids, 190 (2019), pp. 425-439.

[31] V. ThomeE, Galerkin Finite Element Methods for Parabolic Problems, vol. 1054 of Lecture Notes in Mathematics, Springer Berlin Heidelberg, Berlin, 1984.

[32] V. THомéE, Finite difference methods for linear parabolic equations, Handb. Numer. Anal. Vol. I, Elsevier/North-Holland, Amsterdam, (1990), pp. 5-196.

[33] T. XIONG, G. Russo AND J.-M. QIU, Conservative multi-dimensional semi-Lagrangian finite difference scheme: stability and applications to the kinetic and fluid simulations, J. Sci. Comput., 79 (2019), pp. 1241-1270.

[34] N. YI, Y. HUANG AND H. LIU, A conservative discontinuous Galerkin method for nonlinear electromagnetic Schrödinger equations, SIAM J. Sci. Comput., 41 (2019), pp. B1389-B1411.

[35] G. YUAN AND Z. SHENG, Monotone finite volume schemes for diffusion equations on polygonal meshes, J. Comput. Phys., 227 (2008), pp. 6288-6312.

[36] Huasheng Wang, YanPing Chen, Yunding Huang, And Wenting MaO, A posteriori error estimates of the Galerkin spectral methods for space-time fractional diffusion equations, Adv. Appl. Math. Mech., 12 (2020), pp. 87-100.

[37] LiQun Wang, Songming Hou, Liwei Shi, AND PING ZHANG, A bilinear Petrov-Galerkin finite element method for solving elliptic equation with discontinuous coefficients, Adv. Appl. Math. Mech., 11 (2019), pp. 216-240.

[38] Zhiyuan Sun, JUn LiU, AND Pei WANG, A discontinuous Galerkin method by patch reconstruction for convection-diffusion problems, Adv. Appl. Math. Mech., 12 (2020), pp. 729-747.

[39] Shimin CHAI, YONGKUi ZOU AND Wenju ZHAO, A weak Galerkin method with $C^{0}$ element for fourth order linear parabolic equation, Adv. Appl. Math. Mech., 11 (2019), pp. 467-485.

[40] YUAN CHEN, SONGMING HOU, AND XU ZHANG, An immersed finite element method for ellip- 
tic interface problems with multi-domain and triple junction points, Adv. Appl. Math. Mech., 11 (2019), pp. 1005-1021.

[41] SHuANG LiU AND YANPING CHEN, A new two-grid method for expanded mixed finite element solution of nonlinear reaction diffusion equations, Adv. Appl. Math. Mech., 9(3) (2017), pp. 757774 . 